\title{
Unequal Uncertainties and Uncertain Inequalities: An Axiomatic Approach*
}

\author{
Thibault Gajdos`and Eric Maurin ${ }^{\ddagger}$
}

September 2002. Revised July 2003.

\begin{abstract}
In this paper, we provide an axiomatic characterization of social welfare functions for uncertain incomes. Our most general result is that a small number of reasonable assumptions regarding welfare orderings under uncertainty rule out pure ex ante as well as pure ex post evaluations. Any social welfare function that satisfies these axioms should lie strictly between the ex ante and the ex post evaluations of income distributions. We also provide an axiomatic characterization of the weighted average of the minimum and the maximum of ex post and the ex ante evaluations. Journal of Economic Literature Classification Numbers: D31, D63, D81. Key words: Inequality; Uncertainty; Multiple Priors.
\end{abstract}

\section{Introduction}

Consider a society divided into two sectors of equal size - say, sector $A$ and sector $B$. Sector $A$ corresponds to domestic services that cannot be traded at the international level while sector $B$ corresponds to manufacturing industries that can be traded. The government decides if international trade is allowed or not. If no international trade is permitted, whatever happens, wages remain equal to $\$ 1000$ a month in both sectors. In contrast, if international trade is allowed, wages in sector $B$ depend on an exogeneous shock on international demand, which can be positive or negative, with unknown probabilities. If the shock is positive, wages in sector $B$ are $\$ 1500$ a month, whereas if the shock is negative, wages are only $\$ 600$ a month. In other words, trade is assumed to increase simultaneously total income, inequality and uncertainty. The two possible policies can be represented by the following tables.

\begin{tabular}{c|cc} 
no trade & sector $A$ & sector $B$ \\
\hline shock $>0$ & 1000 & 1000 \\
shock $<0$ & 1000 & 1000
\end{tabular}

\begin{tabular}{c|cc} 
trade & sector $A$ & sector $B$ \\
\hline shock $>0$ & 1000 & 1500 \\
shock $<0$ & 1000 & 600
\end{tabular}

${ }^{*}$ We thank Alain Chateauneuf, Michèle Cohen, Marc Fleurbaey, Bernard Salanié, Jean-Marc Tallon and JeanChristophe Vergnaud for useful discussions. We are particularly grateful to an anonymous referee for thorough comments and suggestions.

${ }^{\dagger}$ CNRS-CREST, and ICER, 15 Bd Gabriel Péri, 92245, Malakoff Cedex, France, e-mail: gajdos@ensae.fr

${ }^{\ddagger}$ CREST-Insee and CEPR, 15 Bd Gabriel Péri, 92245 Malakoff Cedex, France, e-mail: maurin@ensae.fr 
The government must decide whether or not to allow international trade. Clearly, the policy which should be chosen depends on the inequality and uncertainty aversions that characterize this particular society. The optimal policy, however, also depends on when individuals' welfare is evaluated, namely before (ex ante) or after (ex post) the resolution of uncertainty. For sufficiently low risk aversion, trade is certainly the best policy ex ante, since it increases the expected earnings in sector $B$ without decreasing them in sector $A$. On the other hand, for sufficiently high inequality aversion, trade is also no doubt the worst policy ex post, since it decreases the lowest wages during bad periods, without increasing them during favorable periods.

More generally, when comparing uncertain income distributions, should we look at the expected income of each person, and consider that the distribution where the inequality of expected incomes is the lowest as the best one? Or should we look at the level of inequalities associated to each possible state of the world, and consider the distribution where the expected level of inequality is the lowest as the best solution?

This problem is not new and has sometimes been labelled as the "timing-effect problem": the outcome of an allocation procedure depends on whether individuals' utility levels are evaluated before or after the resolution of uncertainty. ${ }^{1}$ As stated by Myerson,

"The moral of this story is that simply specifying a social welfare function may not be enough to fully determine a procedure for collective decision making. One must also specify when the individuals' preferences or utility levels should be evaluated; before or after the resolution of uncertainties. The timing of social welfare analysis may make a difference. The timing-effect is often an issue in moral debate, as when people argue about whether a social system should be judged with respect to its actual income distribution or with respect to its distribution of economic opportunities" (p. 884).

To the best of our knowledge, the principles that should be followed to answer this question have not yet been identified in the economic literature. Whereas an extensive body of literature exists on inequality measurement when no uncertainty is involved, very little has been written on inequality measurement under uncertainty, with the important exception of Ben Porath, Gilboa and Schmeidler (1997).

As stated by Ben Porath, Gilboa and Schmeidler (1997), the crucial issue for measuring inequality under uncertainty is to simultaneously take into account the inequality of expected

\footnotetext{
${ }^{1}$ See for instance Broome (1984), Diamond (1967), Myerson (1981) and Hammond (1981), among others, for theoretical work on the timing effect. See Yaari and Bar-Hillel (1984) for empirical evidence about the importance of beliefs in distributional issues.
} 
incomes and the expected inequalities of actual incomes. In this paper, we propose a simple axiomatic characterization of social welfare rankings under uncertainty that captures these two dimensions.

The paper is organized in the following way. Section 2 introduces notation and provides an axiomatic characterization of social welfare functions under uncertainty. Our most general result is that a small number of reasonable assumptions regarding welfare orderings under uncertainty rule out pure ex ante and pure ex post evaluations. Any social welfare function that satisfies these axioms should remain strictly between the ex ante and the ex post evaluations of income distributions. Section 3 provides a reasonable strengthening of our basic axioms which leads to a more complete characterization of admissible social welfare functions. In Section 4, we analyze how the set of welfare functions axiomatized in this paper compares with the family of min-of-means functionals introduced by Ben Porath, Gilboa and Schmeidler (1997). Finally, Section 5 gives our conclusions. All the proofs are gathered in the appendix.

\section{A General Class of Social Preferences Under Uncertainty}

In order to better understand the difficulties raised by uncertainty in evaluating income distributions, let us examine the canonical examples given by Ben Porath, Gilboa and Schmeidler (1997). Consider a society with two individuals, $a$ and $b$, facing two equally likely possible states of the world, $s$ and $t$, and assume that the planner has to choose among the three following social policies, $P_{1}, P_{2}$ and $P_{3}$ :

\begin{tabular}{|c|c|c|c|c|c|c|}
\hline$P_{1}$ & $\begin{array}{ll}a & b\end{array}$ & $P_{2}$ & $a$ & $b$ & $P_{3}$ & $a$ \\
\hline$s$ & $\begin{array}{ll}0 & 0\end{array}$ & $s$ & 1 & 0 & $s$ & 1 \\
\hline$t$ & $\begin{array}{ll}1 & 1\end{array}$ & $t$ & 0 & 1 & $t$ & 1 \\
\hline
\end{tabular}

As argued by Ben Porath, Gilboa and Schmeidler (1997), $P_{2}$ and $P_{3}$ are ex post equivalent, since in both cases, whatever the state of the world, the final income distribution is $(0,1)$ (or $(1,0)$ which, assuming anonymity, is equivalent). On the other hand, $P_{3}$ gives 1 for sure to one individual, and 0 to the other, while $P_{2}$ provides both individuals with the same ex ante income prospects. On these grounds, for a sufficiently low level of uncertainty aversion, it is reasonable to think that $P_{2}$ should be ranked above $P_{3}$. As for $P_{1}$, on the other hand, both individuals face the same income prospects like in $P_{2}$; but in $P_{1}$, there is no ex post inequality, whatever the state of the world. This could lead one to prefer $P_{1}$ over $P_{2}{ }^{2}$

\footnotetext{
${ }^{2}$ As in Gilboa, Ben Porath and Schmeidler (1997), we consider preferences over final allocations: we do not claim that one could not obtain a policy that is strictly preferred to $P_{1}$ by way of ex post transfers among individuals in $P_{2}$.
} 
This example makes clear that there is no hope for providing a reasonable social welfare function over income distributions under uncertainty by simply reducing the problem under consideration to a problem of a choice over uncertain aggregated incomes (say, e.g., by computing a traditional social welfare function $\grave{a}$ la Atkinson-Kolm-Sen in each state, and then reducing the problem to a single decision maker's choice among prospects of welfare). Similarly, reducing the problem by first aggregating individuals' income prospects, and then considering a classical social welfare function defined on these aggregated incomes would not be a reasonable solution. The first procedure would lead us to neglect ex ante considerations and to judge $P_{2}$ and $P_{3}$ as equivalent. In contrast, the second procedure would lead us to neglect ex post considerations and to see $P_{1}$ and $P_{2}$ as equivalent. In other words, these procedures would fail to simultaneously take into account the ex ante and the ex post income distributions.

Ben Porath, Gilboa and Schmeidler (1997) suggest solving this problem by considering a linear combination of the two procedures described above, specifically, a linear combination of the expected Gini index and the Gini index of expected income. This solution captures both ex ante and ex post inequalities. Furthermore, it is a natural generalization of the principles commonly used for evaluating inequality under certainty on the one hand, and for decision making under uncertainty on the other hand. However, the procedure suggested by Ben Porath, Gilboa and Schmeidler (1997) is not the only possible evaluation principle that takes into account both ex ante and ex post inequalities. Any functional that is increasing in both individuals' expected income and snapshot inequalities (say, measured by the Gini index) has the same nice property, provided that it takes its values between the expected Gini and the Gini of the expectation. Furthermore, it is unclear why we should restrict ourselves, as Ben Porath, Gilboa and Schmeidler (1997) did, to decision makers who behave in accordance with the multiple priors model. $^{3}$

There is hence a need for an axiomatic characterization of inequality measurement under uncertainty, which can encompass Ben Porath, Gilboa and Schmeidler's (1997) proposal, and make clear why this specific functional should be used. In this section, we propose a set of axioms which capture what we think to be the basic requirements for any reasonable evaluation of welfare under uncertainty, and identify the corresponding general class of preferences.

\subsection{Notation}

Let $S=\{1, \ldots, s\}$ and $K=\{1, \ldots, n\}$ be respectively a finite set of states of the world, and a finite set of individuals. Let $\mathcal{F}$ denote the set of non-negative real-valued functions on $S \times K$.

\footnotetext{
${ }^{3}$ The multiple priors model assumes that social preferences are concave. It is unclear why preferences over uncertain outcomes should necessarily be concave.
} 
An element $f$ of $\mathcal{F}$ corresponds to a $(s \times n)$ non-negative real-valued matrix. For every $k \geq 0$, we will denote by $\mathbf{k}$ the $(s \times n)$ matrix with all entries equal to $k$.

In this paper, we interpret $\mathcal{F}$ as a set of income distributions under uncertainty. For each $f$ in $\mathcal{F}, f_{\sigma i}$ denotes $i$ 's income if state $\sigma$ occurs, while $f_{\sigma}$. is the row vector that represents the income distribution in state $\sigma$, and $f_{. i}$ the column vector that represents individual $i$ 's income profile. Furthermore, $\mathbf{f}_{\sigma}$. denotes the $(s \times n)$ matrix with all rows equal to $f_{\sigma}$, whereas $\mathbf{f}_{\text {. }}$ denotes the $(s \times n)$ matrix with all columns equal to $f_{\cdot i}$. The set of $\mathbf{f}_{\sigma}$. matrices represents situations where there is no uncertainty: the income distribution is the same in each possible state of the world. In contrast, the $\mathbf{f}_{\cdot i}$ matrices characterize situations where there is no inequality: each individual is faced with the same income prospects.

In the sequel, we adopt the following convention: vectors of $\mathbb{R}_{+}^{n}$ and $\mathbb{R}_{+}^{n+s}$ are considered as row vectors, whereas vectors of $\mathbb{R}_{+}^{s}$ are considered as column vectors. For $\left(x_{1}, \ldots, x_{p}\right),\left(y_{1}, \ldots, y_{p}\right) \in$ $\mathbb{R}^{p},\left(x_{1}, \ldots, x_{p}\right)>\left(y_{1}, \ldots, y_{p}\right)$ means that $x_{i} \geq y_{i}$ for all $i$, and there exists at least one $j$ such that $x_{j}>y_{j}$.

Finally, we use the following definitions. A function $\phi: \mathbb{R}^{q} \rightarrow \mathbb{R}$, with $q \in \mathbb{N}$, is increasing if for all $x, y \in \mathbb{R}^{q}, x>y$ implies $\phi(x)>\phi(y)$. We say that $\phi$ is homogeneous if, for all $\theta>0$, and all $x \in \mathbb{R}^{q}, \phi(\theta x)=\theta \phi(x)$. We say that $\phi$ is homogeneous of degree 0 if for all $\theta>0$, and all $x \in \mathbb{R}^{q}$, $\phi(\theta x)=\phi(x)$. We say that $\phi$ is affine if, for all $x \in \mathbb{R}^{q}$, all $\theta>0$ and all $\eta \in \mathbb{R}, \phi\left(\theta x+\eta \mathbf{1}_{q}\right)=$ $\theta \phi(x)+\eta$, where $\mathbf{1}_{q}$ denotes the unit vector in $\mathbb{R}^{q}$. We say that $\phi$ is a similarity transformation if there exists $\theta>0$ such that $\phi(x)=\theta x$ for all $x \in \mathbb{R}^{q}$. Finally, if $\phi: A \rightarrow B_{\phi}$ and $\psi: A \rightarrow B_{\psi}$ are two functions, $\Im(\phi, \psi)=\left\{(x, y) \in B_{\phi} \times B_{\psi} \mid \exists z \in A\right.$ s.t. $\phi(z)=x$ and $\left.\psi(z)=y\right\}$.

Following the literature on inequality measurement (see, e.g., Atkinson (1970), Kolm (1976) and Sen (1973)), we do not make any assumptions about individuals' preferences. The issue is not to aggregate individuals' preferences, but to propose principles for defining a reasonable collective attitude towards inequality under uncertainty.

\subsection{The structure of social welfare preferences under uncertainty}

We assume that there is a complete, continuous preorder on $\mathcal{F}$. This is the usual basic axiom in the field of normative inequality measurement.

Axiom 1 (ORD) There is a complete, continuous preorder on $\mathcal{F}$, denoted as $\succeq$.

The preorder $\succeq$ can be interpreted as the decision maker's preference relation over $\mathcal{F}$ (one can see this "decision maker" as anybody behind the veil of ignorance). As usual, $\sim$ and $\succ$ will stand for the symmetric and asymmetric part of $\succeq$, respectively. 
Within this framework, we are now going to introduce four axioms which in our view, should be satisfied by any plausible social preference over uncertain income distributions.

The first axiom is a standard monotonicity requirement: if $f$ provides each individual with a higher income than $g$ in each state of the world, then $f$ should be preferred to $g$.

Axiom 2 (MON) For all $f, g$ in $\mathcal{F}$, if $f_{\sigma i}>g_{\sigma i}$ for all $\sigma$ in $S$ and all $i$ in $K$, then $f \succ g$.

Any preorder $\succeq$ on $\mathcal{F}$ naturally induces two preorders $\succeq_{a}$ and $\succeq_{p}$ on $\mathbb{R}_{+}^{s}$ and $\mathbb{R}_{+}^{n}$ respectively, defined as: $f_{\cdot i} \succeq_{a} g_{\cdot i}$ if and only if $\mathbf{f}_{\cdot i} \succeq \mathbf{g}_{\cdot i}$, and $f_{\sigma \cdot} \succeq_{p} g_{\sigma}$. if and only if $\mathbf{f}_{\sigma} . \succeq \mathbf{g}_{\sigma \cdot .}$. The preorder $\succeq_{p}$ captures the decision maker's preferences in the absence of uncertainty, i.e., when the income distribution does not depend on the state of the world. In contrast, $\succeq_{a}$ captures the decision maker's preferences in the absence of inequality, i.e., when each individual faces the same income prospects. In other words $\succeq_{a}$ and $\succeq_{p}$ represent preorders on individual income profiles and snapshot income distributions, respectively.

Let us assume that $f$ and $g$ are such that (a) $f_{\sigma}$. is preferred to $g_{\sigma}$. for all $\sigma$ (with respect to $\succeq_{p}$ ), and (b) $f_{\cdot i}$ is preferred to $g_{\cdot i}$ for all $i$ (with respect to $\succeq_{a}$ ). In other words, $f$ is preferred to $g$ ex post regardless of the state of the world and $f$ is also preferred to $g$ ex ante regardless of the individual on which we focus. In such a case, it is reasonable to assume that $f$ is preferred to $g$ with respect to $\succeq$. This property corresponds to the following axiom of dominance.

Axiom 3 (DOM) Let $f, g \in \mathcal{F}$. If for all $\sigma \in S, f_{\sigma} \succeq_{p} g_{\sigma}$, and for all $i \in K, f_{\cdot i} \succeq_{a} g_{\cdot i}$, then $f \succeq g$. If, moreover, there exists $\sigma \in S$ or $i \in K$ such that $f_{\sigma} \succ_{p} g_{\sigma}$. or $f_{\cdot_{i}} \succ_{a} g_{\cdot i}$, then $f \succ g$.

(DOM) should not be understood as providing a rule for aggregating individuals' preferences. By construction, $\succeq_{a}$ does not represent individuals' preferences but the collective attitude towards uncertainty, exactly as $\succeq_{p}$ represents the collective attitude towards inequality. When these principles imply that (a) any individual is better off in $f$ than in $g$, and (b) any snapshot distribution of $f$ is better than the corresponding snapshot distribution in $g$, then (DOM) simply requires the decision maker to prefer $f$ to $g$.

Now, let us assume that the uncertain income $f_{\sigma i}$ of individual $i$ in state $\sigma$ can be represented as the combination of individual fixed effects that do not depend on the state of the nature, captured by $\lambda_{i}$, on the one hand, and effects that depend on the state of the nature $\mu_{\sigma}$, but that are the same for all individuals, on the other hand. In other words, $f_{\sigma i}=\lambda_{i} \mu_{\sigma}$, for all $i \in K$ and all $\sigma \in S$. In such a case, we can reasonably focus on preorders which satisfy the following property: if the distribution of individual (sure) fixed effects is the same for two matrices $f$ and $g$, but the random variable that generates the variability of individuals' income across states of nature in $f$ is preferred (with respect to $\succeq_{a}$ ) to the one that generates the variability of the 
individuals' income across states of nature in $g$, then $f$ is preferred to $g$. This requirement is formally stated in the following Conditional Dominance Axiom.

Axiom 4 (CDOM) $\forall \lambda \in \mathbb{R}_{+}^{n}, \lambda \neq 0, \mu, \nu \in \mathbb{R}_{+}^{s}, \mu \lambda \succeq \nu \lambda \Leftrightarrow \mu \succeq_{a} \nu$.

Lastly, we will require that $\succeq$ be homogeneous. This axiom is of course debatable; however, this assumption is quite standard in the field of inequality measurement. ${ }^{4}$

Axiom $5(\mathrm{HOM}) \forall f, g \in \mathcal{F}, \forall \theta>0, f \succeq g \Leftrightarrow \theta f \succeq \theta g$.

The following Lemma will prove to be useful in the sequel.

Lemma 1 Assume Axioms (ORD), (MON), (CDOM) and (HOM) hold. There then exists a homogeneous function $I$ which represents $\succeq$, and two homogeneous functions $I_{a}$ and $I_{p}$ which represent $\succeq_{a}$ and $\succeq_{p}$, respectively, such that:

$$
\forall \mu \in \mathbb{R}_{+}^{s}, \lambda \in \mathbb{R}_{+}^{n}, I(\mu \lambda)=I_{a}(\mu) I_{p}(\lambda)
$$

Our first basic finding is that any homogeneous continuous complete social evaluation of the elements of $\mathcal{F}$ that satisfies the dominance and monotonicity axioms introduced in this section should necessarily remain between two very crucial bounds, namely the evaluation of the social welfare distribution before the resolution of uncertainties and the evaluation of the social welfare distribution after the resolution of uncertainty. In order to state this result, we will need the following notation. Following Ben Porath, Gilboa and Schmeidler (1997), for all $f$ in $\mathcal{F}$, and any function $I_{a}: \mathbb{R}_{+}^{s} \rightarrow \mathbb{R}_{+}$and $I_{p}: \mathbb{R}_{+}^{n} \rightarrow \mathbb{R}_{+}$, we will denote by $\left(I_{a} * I_{p}\right)(f)$ the iterative application of $I_{a}$ to the results of $I_{p}$ applied to the rows of $f$, and by $\left(I_{p} * I_{a}\right)(f)$ the iterative application of $I_{p}$ to the results of $I_{a}$ applied to the columns of $f$. $\left(I_{a} * I_{p}\right)$ is hence obtained by first evaluating social welfare in each possible state of the world (through $I_{p}$ ), and then evaluating the distribution of these welfares through $I_{a}$. On the other hand, $\left(I_{p} * I_{a}\right)$ is obtained by first evaluating each individual's welfare by $I_{a}$, and then computing through $I_{p}$ the social value of the distribution of these individual welfares. Formally, we use the following notation: $I_{a}(f)=\left(I_{a}\left(f_{\cdot 1}\right), \ldots, I_{a}\left(f_{\cdot n}\right)\right)$, $I_{p}(f)=\left(I_{p}\left(f_{1}\right), \ldots, I_{p}\left(f_{s}.\right)\right)$, and $\left(I_{a} * I_{p}\right)(f)=I_{a}\left(I_{p}(f)\right),\left(I_{p} * I_{a}\right)(f)=I_{p}\left(I_{a}(f)\right)$. This is a slight abuse in notation, but there is no risk of confusion between $I_{a}\left(f_{\cdot i}\right)\left(I_{p}\left(f_{\sigma \cdot}\right)\right)$, which is a function from $\mathbb{R}_{+}^{s}\left(\mathbb{R}_{+}^{n}\right)$ to $\mathbb{R}_{+}$, and $I_{a}\left(I_{p}\right)$, which is a function from $\mathcal{F}$ to $\mathbb{R}_{+}^{n}\left(\mathbb{R}_{+}^{s}\right)$. Our result then reads as follows.

\footnotetext{
${ }^{4}$ Homogeneity is a potentially problematic property when there is a positive minimum of subsistance.
} 
Theorem 1 Axioms (ORD), (MON), (DOM), (CDOM) and (HOM) are satisfied if, and only if, there exist a continuous, increasing, homogeneous function $I: \mathcal{F} \rightarrow \mathbb{R}_{+}$which represents $\succeq$, two continuous, increasing and homogeneous functions $I_{a}: \mathbb{R}_{+}^{s} \rightarrow \mathbb{R}_{+}$and $I_{p}: \mathbb{R}_{+}^{n} \rightarrow \mathbb{R}_{+}$, which represent $\succeq_{a}$ and $\succeq_{p}$, respectively, and a continuous, increasing and homogeneous function $\Psi: \Im\left(I_{p}, I_{a}\right) \rightarrow \mathbb{R}_{+}$, such that the following hold:

1. $\forall f, g \in \mathcal{F}, f \succeq g \Leftrightarrow I(f)=\Psi\left(I_{p}(f), I_{a}(f)\right) \geq \Psi\left(I_{p}(g), I_{a}(g)\right)=I(g)$

2. If $\left(I_{a} * I_{p}\right)(f)=\left(I_{p} * I_{a}\right)(f)$ then $I(f)=\Psi\left(I_{p}(f), I_{a}(f)\right)=\left(I_{a} * I_{p}\right)(f)$

3. If $\left(I_{a} * I_{p}\right)(f) \neq\left(I_{p} * I_{a}\right)(f)$ then:

$$
\min \left\{\left(I_{a} * I_{p}\right)(f),\left(I_{p} * I_{a}\right)(f)\right\}<I(f)<\max \left\{\left(I_{a} * I_{p}\right)(f),\left(I_{p} * I_{a}\right)(f)\right\} .
$$

Moreover, $\Psi$ is unique given $I_{a}$ and $I_{p}$, and $I_{a}$ and $I_{p}$ are each unique up to a similarity transformation.

The symmetry of the representation theorem might at first sight seem surprising, since Axiom (CDOM) is not symmetric. However, once homogeneity is assumed, (CDOM) implies its symmetric counterpart, as Lemma 1 clearly shows. This is stated formally in the following remark.

Remark 1 Axioms (ORD), (CDOM) and (HOM) imply that for all $\lambda, \hat{\lambda} \in \mathbb{R}_{+}^{n}, \mu \in \mathbb{R}_{+}^{s}, \mu \neq$ $0, \mu \lambda \succeq \mu \hat{\lambda} \Leftrightarrow \lambda \succeq_{p} \hat{\lambda}$

The $I_{a}$ function represents $\succeq_{a}$ and reflects how the decision maker evaluates uncertain income profiles. Symmetrically, the $I_{p}$ function represents $\succeq_{p}$ and captures how the decision maker evaluates income distributions under certainty. Within this framework, $\left(I_{a} * I_{p}\right)$ represents the evaluation through $I_{a}$ of the distribution of ex post social welfares, while $\left(I_{p} * I_{a}\right)$ represents the evaluation through $I_{p}$ of the distribution of ex ante social welfares. These two functionals represent the two key dimensions of social welfare under uncertainty, namely, unequal uncertainties $\left(I_{p} * I_{a}\right)$ and uncertain inequalities $\left(I_{a} * I_{p}\right)$. The first one reflects ex post considerations while the second one only captures ex ante considerations. Theorem 1 shows that under plausible monotonicity and dominance assumptions, a continuous and homogeneous social evaluation cannot correspond to $\left(I_{p} * I_{a}\right)$ or $\left(I_{a} * I_{p}\right)$, but should necessarily remain strictly between these two bounds.

The social welfare functionals defined in Theorem 1 are such that for every $f, I(f)$ is a specific weighted-average of the iterative application of $I_{p}$ to the results of $I_{a}$ and of the iterative 
application of $I_{a}$ to the results of $I_{p}{ }^{5}$ This motivates the following definition of Weighted CrossIterative (WCI) functionals.

Definition 1 A continuous functional $I: \mathcal{F} \rightarrow \mathbb{R}_{+}$is a Weighted Cross-Iterative (WCI) functional, if and only if, there exist two continuous, increasing and homogeneous functions $I_{a}: \mathbb{R}_{+}^{s} \rightarrow \mathbb{R}_{+}$and $I_{p}: \mathbb{R}_{+}^{n} \rightarrow \mathbb{R}_{+}$, a function $\gamma: \mathcal{F} \rightarrow(0,1)$ homogeneous of degree 0 , such that the following hold:

(i) $\forall f \in \mathcal{F}, I(f)=\gamma(f)\left(I_{p} * I_{a}\right)(f)+(1-\gamma(f))\left(I_{a} * I_{p}\right)(f)$

(ii) $\forall f, g \in \mathcal{F},\left(I_{a}(f), I_{p}(f)\right)>\left(I_{a}(f), I_{p}(g)\right) \Rightarrow I(f)>I(g)$.

We denote $\mathcal{W}$ as the set of WCI functionals.

Using Definition 1, Theorem 1 can be restated as follows.

Theorem 2 Axioms (ORD), (MON), (DOM), (CDOM) and (HOM) are satisfied if, and only if, $\succeq$ can be represented by $I \in \mathcal{W}$, with $I_{a}$ and $I_{p}$ representing $\succeq_{a}$ and $\succeq_{p}$, respectively. Moreover, $I_{a}$ and $I_{p}$ are unique up to a similarity transformation, and $\gamma_{\mid\left\{f \in \mathcal{F} \mid\left(I_{a} * I_{p}\right)(f) \neq\left(I_{p} * I_{a}\right)(f)\right\}}$ is unique.

Note that the functionals proposed by Ben Porath, Gilboa and Schmeidler (1997), namely $I(f)=\alpha\left(G_{p} * E_{a}\right)(f)+(1-\alpha)\left(E_{a} * G_{p}\right)(f)$, where $E_{a}$ is the expectation (defined on $\mathbb{R}_{+}^{s}$ ) and $G_{p}$ is a Gini functional (defined on $\mathbb{R}_{+}^{n}$ ), belong to $\mathcal{W}$. Of course, the class of WCI functionals is much larger, since WCI functionals do not necessarily give constant weights to uncertainty in social welfare, on the one hand, and to inequality in uncertain income profiles, on the other hand. Actually, the most striking feature of the functionals proposed by Ben Porath, Gilboa and Schmeidler (1997) is precisely that these relative weights do not depend on the matrix $f$ under consideration (they are always given by the same $\alpha$ and $(1-\alpha)$ ).

Interestingly, Theorem 1 can be used to derive a very fundamental partial ordering over distributions of income under uncertainty: for any $f, g \in \mathcal{F}$, if

$$
\max \left\{\left(I_{a} * I_{p}\right)(f),\left(I_{p} * I_{a}\right)(f)\right\} \leq \min \left\{\left(I_{a} * I_{p}\right)(g),\left(I_{p} * I_{a}\right)(g)\right\}
$$

then $g \succeq f$. If $f$ exhibits both less uncertainty in social welfare and less inequality in uncertain profiles than $g$, then it should be preferred to $g$.

This result provides a very simple means for ranking a wide range of distributions of income under uncertainty. For instance, consider the three social policies $P_{1}, P_{2}$ and $P_{3}$ defined at the

\footnotetext{
${ }^{5}$ To be more specific, for each $f$, there exists $\gamma(f)$ in $(0,1)$, such that $I(f)=\gamma(f)\left(I_{p} * I_{a}\right)(f)+(1-\gamma(f))\left(I_{a} *\right.$ $\left.I_{p}\right)(f)$.
} 
beginning of this section. For the sake of simplicity, assume that $I_{a}$ and $I_{p}$ are symmetric. ${ }^{6}$ Then, if $I_{p}(1,1)=I_{a}(1,1)=1$ (which is only a matter of normalization), we can easily check that $\left(I_{a} * I_{p}\right)\left(P_{1}\right)=\left(I_{p} * I_{a}\right)\left(P_{2}\right)=I_{a}(0,1),\left(I_{a} * I_{p}\right)\left(P_{3}\right)=\left(I_{p} * I_{a}\right)\left(P_{3}\right)=I_{p}(0,1),\left(I_{a} * I_{p}\right)\left(P_{2}\right)=I_{p}(0,1)$ and $\left(I_{p} * I_{a}\right)\left(P_{1}\right)=I_{a}(0,1)$. Therefore, only three cases are possible: $P_{1} \succ P_{2} \succ P_{3}, P_{3} \succ P_{2} \succ P_{1}$ or $P_{1} \sim P_{2} \sim P_{3}$. Which of these orderings holds depends on the relative weight of the inequality and uncertainty aversions. If we assume that $I_{a}$ is the expectation and $I_{p}$ the Gini index, we get $P_{1} \succ P_{2} \succ P_{3}$. This is so because the expectation is neutral towards risk.

\section{$3 \quad$ Weighted Cross-Iterative Functionals}

In this section, we show that a reasonable strengthening of the requirements introduced in the previous section makes it possible to characterize interesting and easy-to-implement sub-classes within the set of WCI functionals. Therefore, hereafter, we assume that $\succeq$ can be represented by a WCI functional.

First, we are going to focus on WCI functionals that satisfy the following strengthening of $(\mathrm{DOM})$, to which we refer to as an Average Dominance Axiom. ${ }^{7}$

Axiom $6(\mathrm{ADOM}) \forall f, g \in \mathcal{F}$, if $\left(I_{a} * I_{p}\right)(f) \geq\left(I_{a} * I_{p}\right)(g)$ and $\left(I_{p} * I_{a}\right)(f) \geq\left(I_{p} * I_{a}\right)(g)$ then $I(f) \geq I(g)$.

This Axiom corresponds to requirements that are clearly stronger than (DOM). Under (ADOM), we do not require uniform ex ante and ex post dominance to prefer $f$ to $g$, but only average dominance. Axiom (ADOM) can be seen as an axiom that imposes some consistency in the principles that rule ex post and ex ante welfare evaluations. To compare two matrices from an ex post viewpoint, we must first evaluate each possible income distribution and then, in a second stage, compare the two sets of social welfare evaluations. Symmetrically, to compare two matrices from an ex ante viewpoint, we must first evaluate income profiles for each individual, and then, in a second stage, compare the two distributions of income profile evaluations. In a sense, axiom (ADOM) says that the principles that rule the first stage of the ex post comparison should be the same as those which rule the second stage of the ex ante comparison, and vice versa. To put it differently, since each possible income distribution is evaluated through $I_{p}$, the distribution of income profiles should also be evaluated through $I_{p}$. Symmetrically, since each individual's income profile is evaluated through $I_{a}$, the social welfare evaluation profiles should also be evaluated through $I_{a}$.

\footnotetext{
${ }^{6}$ The symmetry of $I_{p}$ can be seen as a requirement of impartiality, whereas the symmetry of $I_{a}$ can be justified, in this example, when the two states are equally likely.

${ }^{7}$ Note that for any WCI functional, $I, I_{a}$ and $I_{p}$ are well-defined.
} 
In addition to axiom (ADOM), we will require $\succeq$ to be additive, meaning that adding the same intercept to two matrices does not modify their ranking.

Axiom 7 (ADD) For all $f, g \in \mathcal{F}, \eta \in \mathbb{R}_{+}, f \succeq g \Rightarrow f+\eta \mathbf{1} \succeq g+\eta \mathbf{1}$.

This is a standard assumption regarding social welfare orderings. We could have introduced (ADD) earlier in the text. To be more specific, we could have introduced (ADD) instead of $(\mathrm{HOM})$ in the previous section: substituting (ADD) for (HOM) in the list of axioms used in Theorem 1 leads to the same general class of social preferences (where homogeneity is replaced by unit translatability).

The following theorem characterizes WCI functionals which satisfy (ADOM) and (ADD).

Theorem 3 Suppose that $\succeq$ can be represented by a WCI functional I. Then $\succeq$ satisfies Axioms $(\mathrm{ADOM})$ and $(\mathrm{ADD})$ if, and only if, $I_{a}$ and $I_{p}$ are affine, and there exist $\alpha, \beta \in(0,1)$, such that:

$$
I(f)=\left\{\begin{array}{l}
\alpha\left(I_{p} * I_{a}\right)(f)+(1-\alpha)\left(I_{a} * I_{p}\right)(f), \text { if }\left(I_{a} * I_{p}\right)(f) \geq\left(I_{p} * I_{a}\right)(f) \\
\beta\left(I_{p} * I_{a}\right)(f)+(1-\beta)\left(I_{a} * I_{p}\right)(f), \text { if }\left(I_{p} * I_{a}\right)(f) \geq\left(I_{a} * I_{p}\right)(f) .
\end{array}\right.
$$

Moreover, $\alpha$ and $\beta$ are unique.

The set of such $I$ is denoted $\mathcal{W}_{1}$. Once (ADOM) and (ADD) are satisfied, the weight given to ex ante evaluations only depends on whether they are more important or less important than ex post ones, and vice versa.

Axiom (ADOM) can be strengthened by assuming that the two fundamental dimensions of welfare, namely inequality in uncertainties and uncertainty in inequalities are of commensurate value and equally important. The following axiom of Global Dominance requires that if the best dimension of a matrix $f$ is better than the best dimension of a matrix $g$, and the worst dimension of $f$ is also better than the worst dimension of $g$, then $f$ is better than $g$.

Axiom 8 (GDOM) For all $f, g$ in $\mathcal{F}$, if

$$
\left\{\begin{array}{l}
\max \left\{\left(I_{a} * I_{p}\right)(f),\left(I_{p} * I_{a}\right)(f)\right\} \geq \max \left\{\left(I_{a} * I_{p}\right)(g),\left(I_{p} * I_{a}\right)(g)\right\} \\
\min \left\{\left(I_{a} * I_{p}\right)(f),\left(I_{p} * I_{a}\right)(f)\right\} \geq \min \left\{\left(I_{a} * I_{p}\right)(g),\left(I_{p} * I_{a}\right)(g)\right\}
\end{array}\right.
$$

then, $I(f) \geq I(g)$.

Note that when (GDOM) is satisfied, (ADOM) is also satisfied.

Replacing Axiom (ADOM) by Axiom (GDOM) in Theorem 3 leads to the characterization of the Weighted Max-Min functionals, i.e., of WCI functionals that can be written as a weighted average of the maximum and the minimum of $\left(I_{p} * I_{a}\right)(f)$ and $\left(I_{a} * I_{p}\right)(f)$. 
Theorem 4 Suppose that $\succeq$ can be represented by a WCI functional I. Then $\succeq$ satisfies Axioms $(\mathrm{GDOM})$ and $(\mathrm{ADD})$ if, and only if, $I_{a}$ and $I_{p}$ are affine and there exists $\delta \in(0,1)$, such that:

$$
I(f)=\delta \min \left\{\left(I_{a} * I_{p}\right)(f),\left(I_{p} * I_{a}\right)(f)\right\}+(1-\delta) \max \left\{\left(I_{a} * I_{p}\right)(f),\left(I_{p} * I_{a}\right)(f)\right\} .
$$

Moreover, $\delta$ is unique.

The set of such $I$ is denoted as $\mathcal{W}_{2}$. Once (GDOM) is satisfied, the weights put on the two possible welfare evaluations do not depend on whether they correspond to ex post or ex ante considerations, but only on whether they are the most or the least important. Observe that we have $\mathcal{W}_{2} \subset \mathcal{W}_{1} \subset \mathcal{W}$.

\section{Weighted Cross-Iterative Functionals and Ben-Porath, Gilboa, Schmeidler's proposal}

As noted above, Ben Porath, Gilboa and Schmeidler (1997) have proposed a specific sub-class of WCI functionals, namely the functionals that can be written as $I(f)=\alpha\left(I_{p} * I_{a}\right)(f)+(1-$ $\alpha)\left(I_{a} * I_{p}\right)(f)$, where $I_{a}$ and $I_{p}$ are what they call min-of-means functionals.

Min-of-means functionals are well-known in decision theory under the name of the multiple priors model, and were first introduced by Gilboa and Schmeidler (1989). Special notation is needed in order to define these functionals. Let $\mathcal{P}_{K}$ and $\mathcal{P}_{S}$ be the spaces of probability vectors on $K$ and $S$, respectively. For any $f_{\sigma}$. $\in \mathbb{R}_{+}^{n}$ and $q \in \mathcal{P}_{K}$, let $q \cdot f_{\sigma}=\sum_{i} q_{i} f_{\sigma i}$. Similarly, for any $f_{\cdot i} \in \mathbb{R}_{+}^{s}$, and $q \in \mathcal{P}_{S}$, let $q \cdot f_{\cdot i}=\sum_{\sigma} q_{\sigma} f_{\sigma i}$. Min-of-means functionals are defined as follows.

Definition 2 A functional $I_{a}: \mathbb{R}_{+}^{s} \rightarrow \mathbb{R}_{+}\left(I_{p}: \mathbb{R}_{+}^{n} \rightarrow \mathbb{R}_{+}\right)$is a min-of-means functional if, and only if, there exists a compact and convex subset $\mathcal{C}_{I_{a}}\left(\mathcal{C}_{I_{p}}\right)$ of $\mathcal{P}_{S}\left(\mathcal{P}_{K}\right)$, such that for all $f_{\cdot i} \in \mathbb{R}_{+}^{s}$ $\left(f_{\sigma \cdot} \in \mathbb{R}_{+}^{n}\right), I_{a}\left(f_{\cdot i}\right)=\min _{q \in \mathcal{C}_{I_{a}}} q \cdot f_{\cdot i}\left(I_{p}\left(f_{\sigma \cdot}\right)=\min _{q \in \mathcal{C}_{I_{p}}} q \cdot f_{\sigma \cdot}\right)$.

The class of functionals $\mathcal{W}_{3}$ proposed by Ben-Porath, Gilboa and Schmeidler can then be defined as:

$$
\mathcal{W}_{3}=\left\{I \in \mathcal{W} \mid I_{a}, I_{p} \text { are min-of-means functionals and } \forall f, g \in \mathcal{F}, \gamma(f)=\gamma(g)\right\}
$$

Any functional in $\mathcal{W}_{3}$ clearly belongs to $\mathcal{W}_{1}$, meaning $\mathcal{W}_{3} \subset \mathcal{W}_{1}$. In contrast, elements of $\mathcal{W}_{3}$ do not necessarily satisfy (GDOM), and there exist functionals in $\mathcal{W}_{3}$ which do not belong to $\mathcal{W}_{2}$ (i.e., $\mathcal{W}_{3} \not \subset \mathcal{W}_{2}$ ).

Any $I$ in $\mathcal{W}_{3}$ gives the same weight to ex ante inequalities regardless of whether they are more or less important than ex post ones and vice versa. In contrast, any $I$ in $\mathcal{W}_{2}$ systematically puts more emphasis on the dominant source of inequality. 
Before exploring the relationship between these two classes of functionals, it may be useful to define a special subset of the set of min-of-means functionals, namely the generalized minimum operators. We say that a min-of-means functional $I_{a}: \mathbb{R}_{+}^{s} \rightarrow \mathbb{R}_{+}$is a generalized minimum operator if there exist $k \in S$ and $S_{0}=\left\{\sigma_{1}, \ldots, \sigma_{k}\right\} \subseteq S$ such that for all $\mu=\left(\mu_{1}, \ldots, \mu_{s}\right) \in \mathbb{R}_{+}^{s}$, $I_{a}(\mu)=\min _{\sigma \in S_{0}} \mu_{\sigma}$. Similarly, $I_{p}: \mathbb{R}_{+}^{n} \rightarrow \mathbb{R}_{+}$is a generalized minimum operator if there exist $k \in K$ and $K_{0}=\left\{i_{1}, \ldots, i_{k}\right\} \subseteq K$ such that for all $\lambda=\left(\lambda_{1}, \ldots, \lambda_{n}\right) \in \mathbb{R}_{+}^{n}, I_{p}(\lambda)=\min _{i \in K_{0}} \lambda_{i}$.

The following theorem examines the conditions under which an element of $\mathcal{W}_{3}$ satisfies the axioms introduced in this paper and belongs to $\mathcal{W}_{2}$.

Theorem 5 Assume $I \in \mathcal{W}_{3}$. Then, $I \in \mathcal{W}_{2}$ if and only if at least one of the two following conditions is satisfied:

(i) $\forall f \in \mathcal{F}, I(f)=\frac{1}{2}\left(I_{p} * I_{a}\right)(f)+\frac{1}{2}\left(I_{a} * I_{p}\right)(f)$

(ii) At least one of $I_{a}$ and $I_{p}$ is either (a) a mathematical expectation with respect to a given probability measure or (b) a generalized minimum operator.

A potentially interesting subclass of $\mathcal{W}_{3}$ is the set of symmetric min-of-means (i.e., such that for all $f \in \mathcal{F}, I(f)=I\left(f^{\prime}\right)$ for all $f^{\prime}$ such that $f^{\prime}$ is obtained by a permutation of the rows and the columns of $f$ ). The symmetry assumption may be relevant whenever the states of the world are equally likely. Given that the only symmetric generalized minimum operator is the minimum operator (on all the components) and that the only symmetric probability vector is the uniform one (i.e., the vector whose all components are equal), Theorem 5 has the following corollary.

Corollary 1 Assume $I \in \mathcal{W}_{3}$ and $I$ is symmetric. Then $I \in \mathcal{W}_{2}$ if and only if at least one of the two following conditions is satisfied:

(i) $\forall f \in \mathcal{F}, I(f)=\frac{1}{2}\left(I_{p} * I_{a}\right)(f)+\frac{1}{2}\left(I_{a} * I_{p}\right)(f)$

(ii) At least one of $I_{a}$ and $I_{p}$ is either (a) the mathematical expectation with respect to the uniform distribution or (b) the minimum operator.

Generally speaking, once we exclude the specific cases of risk (or inequality) neutrality and extreme egalitarianism (or extreme aversion to risk), the only functionals that belong simultaneously to $\mathcal{W}_{2}$ and $\mathcal{W}_{3}$ are the arithmetic means of ex ante and ex post social welfare evaluation (through min-of-means). The key feature of these functionals is that any given shifts in ex post levels of social welfare can actually be compensated by symmetric shifts in ex ante levels of individual welfares, i.e., by shifts whose social evaluation is the same as the evaluation of the $e x$ post shifts in absolute value. 
To make this property explicit, let us define, for each vector $u$ in $\mathbb{R}_{+}^{n}$, the set $\mathcal{S}(u)$ of vectors $v$ of $\mathbb{R}_{+}^{s}$, such that for some constant $k>0$, the matrix $\mathbf{u}_{\sigma} .+\mathbf{k}$ with all rows equal to $u+k \mathbf{1}_{n}$ (i.e., a matrix with no uncertainty, where only inequality matters) is equivalent to the matrix $\mathbf{v}_{i}+\mathbf{k}$ with all columns equal to $v+k \mathbf{1}_{s}$ (i.e., a matrix with no inequality, where only uncertainty matters). ${ }^{8}$ Formally:

$$
\mathcal{S}(u)=\left\{v \in \mathbb{R}^{s} \mid \exists k>0, \text { s.t. } \mathbf{V}_{\cdot i}+\mathbf{k} \sim \mathbf{u}_{\sigma}+\mathbf{k}\right\} .
$$

Then, for any matrix $f \in \mathcal{F}$, one can define the set $\mathcal{E}(f) \subseteq \mathcal{F}$ of matrices that are obtained from $f$ by shifts in ex post levels of social welfare and shifts in ex ante levels of individual welfares, whose social evaluations are the same. Formally,

$$
\mathcal{E}(f)=\left\{g \in \mathcal{F} \mid \exists u \in \mathbb{R}^{n}, v \in \mathcal{S}(u) \text {, s.t. }\left(I_{a}(g), I_{p}(g)\right)=\left(I_{a}(f)-u, I_{p}(f)+v\right)\right\} .
$$

We can now state formally the desired Axiom of symmetry.

Axiom $9(\mathrm{SYM}) \forall f \in \mathcal{F}, g \in \mathcal{E}(f) \Rightarrow f \sim g$.

As it turns out, the preorder $\succeq$ can be represented by a WCI functional and satisfies Axioms (ADD) and (SYM) if, and only if, it can be represented by the arithmetic mean of ex ante and ex post welfare evaluations.

Theorem 6 Suppose that $\succeq$ can be represented by a WCI functional I. Then $\succeq$ satisfies Axioms $(A D D)$ and $(S Y M)$ if, and only if, $I_{a}$ and $I_{p}$ are affine, and:

$$
I(f)=\frac{1}{2}\left(I_{p} * I_{a}\right)(f)+\frac{1}{2}\left(I_{a} * I_{p}\right)(f) .
$$

\section{Conclusion}

In this paper, we show that under some reasonable monotonicity and dominance assumptions, any continuous homogeneous social welfare function should lie strictly between the ex ante and the ex post evaluations of income distributions. We propose the weighted average of the minimum and the maximum of ex post and ex ante evaluations as a new means for evaluating welfare under uncertainty.

Clearly, this new evaluation tool can be used in a potentially very large set of contexts. The usual practice is to rank public policies according to their impact on either the observed distribution of income or on the distribution of expected income. Once we do not neglect macroeconomic

\footnotetext{
${ }^{8}$ We denote by $k_{\sigma}$. the vector of $\mathbb{R}_{+}^{n}$ with all entries equal to $k$, and by $k_{\cdot i}$ the vector of $\mathbb{R}_{+}^{s}$ with all entries equal to $k$.
} 
uncertainty, we should not rely on either pure ex ante or pure ex post considerations, but on one of the mixtures that are axiomatized in this paper.

At a very general level, our paper can be understood as an attempt to evaluate income distributions when it is not indifferent whether income varies across states of the world or across individuals. We think that this approach could be generalized to any problem of welfare evaluation where the sources of income variability matter. One such problem is the evaluation of income distributions according to the principle of equality of opportunity. This principle requires giving different weights to inequalities generated by circumstances beyond the control of individuals on the one hand, and on the other hand, to inequalities generated by actions that reflect individuals' own free volition. We speculate that the axiomatization and design of new means for implementing this principle can be obtained following a very similar route as the one used in this paper. This issue is part of our research agenda. 


\section{Appendix}

\section{Proof of Lemma 1.}

By Debreu (1959), Axiom (ORD) implies that there exists a continuous function $I: \mathcal{F} \rightarrow \mathbb{R}$ representing $\succeq$. We can, therefore, define $I_{a}$ and $I_{p}$, representing $\succeq_{a}$ and $\succeq_{p}$, respectively, as follows: $I_{a}\left(f_{\cdot i}\right)=I\left(\mathbf{f}_{\cdot \mathbf{i}}\right)$, and $I_{p}\left(f_{\sigma \cdot}\right)=I\left(\mathbf{f}_{\sigma \cdot}\right)$, for all $f_{\cdot i} \in \mathbb{R}_{+}^{s}$ and $f_{\sigma} \cdot \in \mathbb{R}_{+}^{n}$. Furthermore, we can, without loss of generality, normalize $I$ such that $I(\mathbf{1})=1$, where $\mathbf{1}$ is the matrix in $\mathcal{F}$ whose elements are all equal to 1 . Axiom (HOM) implies that $I$ can be chosen to be homogeneous, from which it follows that $I_{a}$ and $I_{p}$ are homogeneous too. Moreover, by continuity of $I, I(\mathbf{0})=0$, where $\mathbf{0}$ is the $(s \times n)$ matrix with all entries equal to 0 . Therefore, by Axiom (MON), $I(f) \geq 0$ for all $f$ in $\mathcal{F}$. This obviously implies that $I_{a}(\mu) \geq 0$ for all $\mu \in \mathbb{R}_{+}^{s}$, and $I_{p}(\lambda) \geq 0$ for all $\lambda \in \mathbb{R}_{+}^{n}$.

Let $f=\mu \lambda \in \mathcal{F}$, with $\mu \in \mathbb{R}_{+}^{s}$ and $\lambda \in \mathbb{R}_{+}^{n}, \lambda \neq 0$. Define $g$ by: $g_{\sigma i}=I_{a}(\mu) \lambda_{i}$, for all $\sigma \in S$ and all $i \in K$. Observe that $g=\nu \lambda$, with $\nu=\left(I_{a}(\mu), \ldots, I_{a}(\mu)\right) \in \mathbb{R}_{+}^{s}$. By homogeneity of $I_{a}$, and given the normalization choice $I(\mathbf{1})=1$, we have: $I_{a}(\nu)=I_{a}(\mu)$. Therefore, by Axiom (CDOM), we have $f \sim g$, i.e., $I(f)=I(g)$. But, by homogeneity of $I, I(g)=I_{a}(\mu) I\left(\mathbf{h}_{\sigma}\right.$.), with $h_{\sigma}=\lambda$ for every $\sigma$. Since, by definition of $I_{p}, I\left(\mathbf{h}_{\sigma \cdot}\right)=I_{p}(\lambda)$, we get: $I(g)=I_{a}(\mu) I_{p}(\lambda)=I(f)$, the desired result.

Proof of Theorem 1.

First, we prove the "only if" part of the Theorem.

Claim 1. Axioms (ORD), (MON), (DOM) and (HOM) imply that there exist a continuous, increasing, homogeneous function I which represents $\succeq$, two continuous, increasing and homogeneous functions $I_{a}: \mathbb{R}_{+}^{s} \rightarrow \mathbb{R}_{+}$and $I_{p}: \mathbb{R}_{+}^{n} \rightarrow \mathbb{R}_{+}$, which represent $\succeq_{a}$ and $\succeq_{p}$, respectively, a continuous, increasing and homogeneous function $\Psi: \Im\left(I_{p}, I_{a}\right) \rightarrow \mathbb{R}_{+}$, such that $\forall f, g \in \mathcal{F}$ :

$$
f \succeq g \Leftrightarrow I(f)=\Psi\left(I_{p}(f), I_{a}(f)\right) \geq \Psi\left(I_{p}(g), I_{a}(g)\right)=I(g) .
$$

Proof.

By Debreu (1959), Axiom (ORD) holds if, and only if, there exists a continuous function $I: \mathcal{F} \rightarrow \mathbb{R}$ such that $I$ represents $\succeq$. Furthermore, Axiom (MON) implies that $I$ is increasing.

Without loss of generality, we can choose $I$ such that $I(\mathbf{1})=1$. Axiom (HOM) implies that $I$ can be chosen to be homogeneous, i.e., such that $I(\theta f)=\theta I(f)$ for all $\theta>0$ and $f \in \mathcal{F}$. The homogeneity and the continuity of $I$ imply that $I(\mathbf{0})=0$. Therefore, by Axiom (MON), $I(f) \geq 0$ for all $f$ in $\mathcal{F}$, i.e., $I$ takes its values in $\mathbb{R}_{+}$. 
Considering the restriction of $I$ on sets of matrices $\mathbf{f}_{\sigma}$. and $\mathbf{f}_{\text {. }}$ respectively, Axiom (ORD) implies that there exist two non-negative continuous functions $I_{p}$ and $I_{a}$ representing $\succeq_{p}$ and $\succeq_{a}$ respectively, and that these functions are increasing and homogeneous, since $I$ is.

Now, for any $x \in \Im\left(I_{p}, I_{a}\right)$, let $\Gamma(x)=\left\{f \in \mathcal{F} \mid\left(I_{p}(f), I_{a}(f)\right)=x\right\} .{ }^{9}$ Axiom (DOM) implies that if two matrices $f$ and $g$ are such that $I_{p}(f)=I_{p}(g)$ and $I_{a}(f)=I_{a}(g)$, then $f \sim g$, and therefore $I(f)=I(g)$. Hence, for all $x \in \Im\left(I_{p}, I_{a}\right)$, and all $f, g \in \Gamma(x), I(f)=I(g)$. Now define $\Psi: \Im\left(I_{p}, I_{a}\right) \rightarrow \mathbb{R}_{+}$by: $\Psi(x)=\left.I(f)\right|_{f \in \Gamma(x)}$. For any $f \in \mathcal{F}, I(f)=\Psi\left(I_{p}(f), I_{a}(f)\right)$.

Using (DOM) and the fact that $I_{a}$ and $I_{p}$ are homogenous, it is straightforward to show that $\Psi$ is homogenous and increasing too. We now prove the continuity of $\Psi$. Let $x^{(k)} \in \Im\left(I_{p}, I_{a}\right)$, be a sequence such that $\lim _{k \rightarrow \infty} x^{(k)}=x \in \Im\left(I_{p}, I_{a}\right)$. We are going to show that $\lim _{k \rightarrow \infty} \Psi\left(x^{(k)}\right)=$ $\Psi(x)$.

Let $f^{(k)}$ be a sequence such that $f^{(k)} \in \Gamma\left(x^{(k)}\right)$ for all $k$, and $f \in \Gamma(x)$. Given that $\lim _{k \rightarrow \infty} x^{(k)}=x$, for any $\varepsilon>0$ there exists $N \in \mathbb{N}$ such that, for any $k>N$,

$$
(1-\varepsilon) x<x^{(k)}<(1+\varepsilon) x
$$

Thus, given that $I_{a}$ and $I_{p}$ are homogenous, we have:

$$
\left(I_{p}((1-\varepsilon) f), I_{a}((1-\varepsilon) f)\right)<\left(I_{p}\left(f^{(k)}\right), I_{a}\left(f^{(k)}\right)\right)<\left(I_{p}((1+\varepsilon) f), I_{a}((1+\varepsilon) f)\right),
$$

and therefore by Axiom (DOM):

$$
I((1-\varepsilon) f)<I\left(f^{(k)}\right)<I((1+\varepsilon) f)
$$

Finally, homogeneity of $I$ implies: $(1-\varepsilon) I(f)<I\left(f^{(k)}\right)<(1+\varepsilon) I(f)$, which implies that $\lim _{k \rightarrow \infty} I\left(f^{(k)}\right)=I(f)$. Thus, $\lim _{k \rightarrow \infty} \Psi\left(x^{(k)}\right)=\Psi(x)$. $\diamond$

Claim 2. Axioms (ORD), (MON), (DOM), (CDOM) and (HOM) imply that, for all $f, g \in \mathcal{F}$ such that $\left(I_{a} * I_{p}\right)(f) \neq\left(I_{p} * I_{a}\right)(f)$,

$$
\min \left\{\left(I_{a} * I_{p}\right)(f),\left(I_{p} * I_{a}\right)(f)\right\}<I(f)<\max \left\{\left(I_{a} * I_{p}\right)(f),\left(I_{p} * I_{a}\right)(f)\right\}
$$

Proof.

Let $f \in \mathcal{F}$, with $f \neq \mathbf{0}$ and define $g$ and $h$ as follows: $g_{\sigma i}=\frac{I_{a}\left(f_{\cdot i}\right) I_{p}\left(f_{\sigma \cdot}\right)}{\left(I_{a} * I_{p}\right)(f)}$ and $h_{\sigma i}=\frac{I_{a}\left(f_{\cdot i}\right) I_{p}\left(f_{\sigma \cdot}\right)}{\left(I_{p} * I_{a}\right)(f)}$, for all $\sigma$ in $S$ and all $i$ in $K .{ }^{10}$ Observe that, since $f \neq \mathbf{0}$, Axiom (MON) implies that $g$ and $h$ are well defined.

\footnotetext{
${ }^{9}$ Recall that $\Im\left(I_{p}, I_{a}\right)=\left\{z \in \mathbb{R}_{+}^{s+n} \mid \exists f \in \mathcal{F},\left(I_{p}(f), I_{a}(f)\right)=z\right\}$.

${ }^{10}$ Observe that $I(\mathbf{0})=\left(I_{a} * I_{p}\right)(\mathbf{0})=\left(I_{p} * I_{a}\right)(\mathbf{0})=0$, and therefore, condition $(2)$ of the Theorem is obviously satisfied in this case.
} 
First, let us assume that $\left(I_{a} * I_{p}\right)(f)<\left(I_{p} * I_{a}\right)(f)$. We get: $I_{a}\left(g_{\cdot i}\right)=\frac{I_{a}\left(f_{\cdot}\right)}{\left(I_{a} * I_{p}\right)(f)}\left(I_{a} * I_{p}\right)(f)$ for all $i$ in $K$ by homogeneity of $I_{a}$. Therefore: $I_{a}\left(g_{\cdot i}\right)=I_{a}\left(f_{\cdot}\right)$ for all $i$ in $K$. On the other hand, $I_{p}\left(g_{\sigma}\right)=\frac{I_{p}\left(f_{\sigma \cdot}\right)}{\left(I_{a} * I_{p}\right)(f)}\left(I_{p} * I_{a}\right)(f)$ for all $i$ in $K$, by homogeneity of $I_{p}$, which implies $I_{p}\left(g_{\sigma \cdot}\right)>I_{p}\left(f_{\sigma}\right)$ for all $\sigma$ in $S$, since $\left(I_{a} * I_{p}\right)(f)<\left(I_{p} * I_{a}\right)(f)$. Therefore, it follows from Claim 1 that $g \succ f$.

Observe that $g=\frac{1}{\left(I_{a} * I_{p}\right)(f)} \mu_{1} \lambda_{1}$, with $\lambda_{1}=\left(I_{a}\left(f_{\cdot 1}\right), \ldots, I_{a}\left(f_{\cdot n}\right)\right)$ and $\mu_{1}=\left(I_{p}\left(f_{1} \cdot\right), \ldots, I_{p}\left(f_{s \cdot}\right)\right)$. Therefore, by homogeneity of $I$, and using Lemma 1 , we have $I(g)=\frac{1}{\left(I_{a} * I_{p}\right)(f)} I_{a}\left(\mu_{1}\right) I_{p}\left(\lambda_{1}\right)$. By definition, $I_{a}\left(\mu_{1}\right)=\left(I_{a} * I_{p}\right)(f)$, and $I_{p}\left(\lambda_{1}\right)=\left(I_{p} * I_{a}\right)(f)$. Therefore, $I(g)=\left(I_{p} * I_{a}\right)(f)$, which implies: $I(f)<\left(I_{p} * I_{a}\right)(f)$.

On the other hand, $I_{a}\left(h_{\cdot i}\right)=\frac{I_{a}\left(f_{\cdot}\right)}{\left(I_{p} * I_{a}\right)(f)}\left(I_{a} * I_{p}\right)(f)$ for all $i$ in $K$, by homogeneity of $I_{a}$. Therefore, $I_{a}\left(h_{\cdot i}\right)<I_{a}\left(f_{\cdot i}\right)$ for all $i$ in $K$ since $\left(I_{a} * I_{p}\right)(f)<\left(I_{p} * I_{a}\right)(f)$, and $I_{p}\left(h_{\sigma \cdot}\right)=$ $\frac{I_{p}\left(f_{\sigma \cdot}\right)}{\left(I_{p} * I_{a}\right)(f)}\left(I_{p} * I_{a}\right)(f)$ for all $i$ in $K$, by homogeneity of $I_{p}$, which implies $I_{p}\left(h_{\sigma \cdot}\right)=I_{p}\left(f_{\sigma}\right)$ for all $\sigma$ in $S$. Therefore, it follows from Claim 1 that $f \succ h$.

By homogeneity of $I, I(h)=\frac{1}{\left(I_{p} * I_{a}\right)(f)} I\left(\lambda_{1} \mu_{1}\right)$. Therefore, using again Lemma 1, we get: $I(h)=\left(I_{a} * I_{p}\right)(f)$. Therefore, $I(f)>\left(I_{a} * I_{p}\right)(f)$, from which it follows that $\left(I_{a} * I_{p}\right)(f)<$ $I(f)<\left(I_{p} * I_{a}\right)(f)$.

Using a symmetrical argument, we can show that if $\left(I_{a} * I_{p}\right)(f)>\left(I_{p} * I_{a}\right)(f)$, then $\left(I_{p} *\right.$ $\left.I_{a}\right)(f)<I(f)<\left(I_{a} * I_{p}\right)(f) . \diamond$

Claim 3. Axioms (ORD), (MON), (DOM), (CDOM) and (HOM) imply that for all $f \in \mathcal{F}$,

$$
\left(I_{a} * I_{p}\right)(f)=\left(I_{p} * I_{a}\right)(f) \Rightarrow I(f)=\Psi\left(I_{p}(f), I_{a}(f)\right)=\left(I_{a} * I_{p}\right)(f)
$$

Proof.

Assume that $\left(I_{a} * I_{p}\right)(f)=\left(I_{p} * I_{a}\right)(f)$. Using the same notation as in Claim 2, we clearly get that $f \sim g \sim h$ and therefore, $\left(I_{a} * I_{p}\right)(f)=\left(I_{p} * I_{a}\right)(f)=I(f) . \diamond$

Claim 4. $I_{a}$ and $I_{p}$ are unique up to a similarity transformation.

Proof.

Due to the symmetry of the problem, we will focus on $I_{a}$ (the proof for $I_{p}$ is similar).

Let us assume that there exist two homogeneous functionals $I_{a}$ and $\hat{I}_{a}$ that represent $\succeq_{a}$. Let $\tilde{I}_{a}=\frac{I_{a}(1, \ldots, 1)}{\hat{I}_{a}(1, \ldots, 1)} \hat{I}_{a}$. Then, $\tilde{I}_{a}(1, \ldots, 1)=I_{a}(1, \ldots, 1)$. Assume there exists $\mu \in \mathbb{R}_{+}^{s}$ such that $I_{a}(\mu) \neq \tilde{I}_{a}(\mu)$. Without loss of generality, let $\tilde{I}_{a}(\mu)=\xi>I_{a}(\mu)=\zeta$.

Let us consider $\mu_{1}=\left(\frac{\xi}{I_{a}(1, \ldots, 1)}, \ldots, \frac{\xi}{I_{a}(1, \ldots, 1)}\right)$. By definition of $\tilde{I}_{a}, \tilde{I}_{a}\left(\mu_{1}\right)=\frac{I_{a}(1, \ldots, 1)}{\tilde{I}_{a}(1, \ldots, 1)} \hat{I}_{a}\left(\mu_{1}\right)$. The homogeneity of $\hat{I}_{a}$ then implies: $\tilde{I}_{a}\left(\mu_{1}\right)=\xi$. Therefore, $\mu \sim_{a} \mu_{1}$.

Similarly, let us define $\mu_{2}=\left(\frac{\zeta}{I_{a}(1, \ldots, 1)}, \ldots, \frac{\zeta}{I_{a}(1, \ldots, 1)}\right)$. Using Axiom (HOM) again, one gets $I_{a}\left(\mu_{2}\right)=\zeta$. Hence, $\mu_{2} \sim_{a} \mu$. 
Since $\mu_{1} \sim_{a} \mu$ and $\mu_{2} \sim_{a} \mu$, we finally get $\mu_{1} \sim_{a} \mu_{2}$, which contradicts the increasingness of $\succeq_{a}$, since $\xi>\zeta . \diamond$

Claim 5. Given $I_{a}$ and $I_{p}, \Psi$ is unique up to a similarity transformation.

Proof.

Assume that there exist two homogeneous functionals $\Psi_{1}$ and $\Psi_{2}$ such that $\Psi_{1}\left(I_{a}, I_{p}\right)$ and $\Psi_{2}\left(I_{a}, I_{p}\right)$ both represent $\succeq$. Since $I_{a}$ and $I_{p}$ are defined up to a similarity transformation, we can assume without loss of generality that $I_{a}(1, \ldots, 1)=I_{p}(1, \ldots, 1)=1$. Let $\Psi_{3}=\frac{\Psi_{1}(1, \ldots, 1)}{\Psi_{2}(1, \ldots, 1)} \Psi_{2}$. Then, $\Psi_{3}(1, \ldots, 1)=\Psi_{1}(1, \ldots, 1)$. Assume there exists $f$ in $\mathcal{F}$ such that $\Psi_{3}\left(I_{a}(f), I_{p}(f)\right) \neq$ $\Psi_{1}\left(I_{a}(f), I_{p}(f)\right)$. Without loss of generality, let $\Psi_{3}\left(I_{a}(f), I_{p}(f)\right)=\xi>\Psi_{1}\left(I_{a}(f), I_{p}(f)\right)=\zeta$.

Now, define $g$ as follows: $g_{\sigma i}=\frac{\xi}{\Psi_{1}(1, \ldots, 1)}$ for all $\sigma$ and all $i$. By homogeneity of $\Psi_{3}$, and given the normalization of $I_{a}$ and $I_{p}, \Psi_{3}\left(I_{a}(g), I_{p}(g)\right)=\xi$. Therefore, $g \sim f$.

Similarly, let $h$ be defined by: $h_{\sigma i}=\frac{\zeta}{\Psi_{1}(1, \ldots, 1)}$ for all $\sigma$ and all $i$. By homogeneity of $\Psi_{1}$, and given the normalization of $I_{a}$ and $I_{p}, \Psi_{1}\left(I_{a}(f), I_{p}(f)\right)=\zeta$. Therefore, $h \sim f$.

Since $g \sim f$ and $h \sim f$, we finally get $g \sim f$, which contradicts Axiom (MON), since $\xi>\zeta$. Hence, $\Psi_{3}=\Psi_{1}$. Therefore, $\Psi$ is unique up to a similarity transformation.

We will now turn to the "if" part of the Theorem.

Axiom (ORD) is obviously satisfied. Since $\Psi, I_{a}$ and $I_{p}$ are homogeneous, Axiom (HOM) is satisfied. Furthermore, since $\Psi$ is increasing, Axiom (DOM) holds, and since $I_{a}$ and $I_{p}$ are increasing, Axiom (MON) holds too.

Now, let $f=\mu \lambda$ and $g=\nu \lambda$ as in Axiom (CDOM), with $\mu \succeq_{a} \nu$. Homogeneity of $I_{a}$ and $I_{p}$ imply $I_{a}\left(f_{\cdot i}\right)=I_{a}(\mu) \lambda_{i}$ for all $i \in K$ and $I_{p}\left(f_{\sigma \cdot}\right)=I_{p}(\lambda) \mu_{\sigma}$ for all $\sigma \in S$. Therefore, by homogeneity of $I_{a}$ and $I_{p}$, we have $\left(I_{a} * I_{p}\right)(f)=\left(I_{p} * I_{a}\right)(f)=I_{a}(\mu) I_{p}(\lambda)$. Hence, by condition (1) in the Theorem, it follows that $I(f)=I_{a}(\mu) I_{p}(\lambda)$. Similarly, $I(g)=I_{a}(\nu) I_{p}(\lambda)$. Observe that $I_{p}(\lambda)>0$, because $\lambda \neq 0$ and $I_{p}$ is homogeneous and increasing. Therefore, $I(f) \geq I(g)$, if and only if, $I_{a}(\mu) \geq I_{a}(\nu)$, i.e., $\mu \succeq_{a} \nu$ : Axiom (CDOM) is hence satisfied.

Finally, any similarity transformation of $I_{a}$ and $I_{p}$ also leads to a functional representing $\succeq$ (with $\Psi$ being appropriately adjusted), which completes the proof.

\section{Proof of Theorem 2.}

Assume that $I$ satisfies the conditions of Theorem 2. Then, for all $f$ in $\mathcal{F}$, we can define, using Theorem 1:

$$
\left\{\begin{array}{l}
\gamma(f)=\frac{I(f)-\left(I_{a} * I_{p}\right)(f)}{\left(I_{p} * I_{a}\right)(f)-\left(I_{a} * I_{p}\right)(f)} \text { if }\left(I_{p} * I_{a}\right)(f) \neq\left(I_{a} * I_{p}\right)(f) \\
\gamma(f)=\frac{1}{2} \text { if }\left(I_{p} * I_{a}\right)(f)=\left(I_{a} * I_{p}\right)(f)
\end{array}\right.
$$


Clearly $\gamma(f)$ belongs to $(0,1)$, is homogenous of degree zero and satisfies,

$$
I(f)=\gamma(f)\left(I_{p} * I_{a}\right)(f)+(1-\gamma(f))\left(I_{a} * I_{p}\right)(f), \forall f \in \mathcal{F} .
$$

Furthermore, condition (1) in Theorem 1 and the requirement that $\Psi$ be increasing imply that for all $f, g$ in $\mathcal{F}$, such that $\left(I_{a}(f), I_{p}(f)\right)>\left(I_{a}(f), I_{p}(g)\right), I(f)>I(g)$, i.e., condition $(i i)$ of Definition 1 is satisfied. Therefore, if $I$ satisfies the conditions of Theorem 2, it can be written as a WCI functional.

Uniqueness up to a similarity transformation of $I_{a}$ and $I_{p}$ are proven as in Theorem 1 . The uniqueness of $\gamma_{\mid\left\{f \in \mathcal{F} \mid\left(I_{a} * I_{p}\right)(f) \neq\left(I_{p} * I_{a}\right)(f)\right\}}$ is straightforward.

Conversely, any WCI functional with $I_{a}$ and $I_{p}$ (representing respectively $\succeq_{a}$ and $\succeq_{p}$ ), obviously satisfies the conditions imposed on $I$ in Theorem 1.

\section{Proof of Theorem 3.}

We first prove the "only if" part of the Theorem.

By definition, if $I$ is a WCI, there exists a function $\gamma: \mathcal{F} \rightarrow(0,1)$ homogeneous of degree 0 , and two homogeneous increasing functions $I_{a}$ and $I_{p}$, which represent respectively $\succeq_{a}$ and $\succeq_{p}$, such that $\succeq$ can be represented by $I(f)=\gamma(f)\left(I_{p} * I_{a}\right)(f)+(1-\gamma(f))\left(I_{a} * I_{p}\right)(f)$. Without loss of generality, we can normalize $I$ such that $I(\mathbf{1})=1$.

The proof goes through three steps.

Claim 1. $\forall f \in \mathcal{F}$ such that $\left(I_{p} * I_{a}\right)(f) \neq\left(I_{a} * I_{p}\right)(f), \forall \theta>0, \forall \eta \in \mathbb{R}_{+}, \gamma(\theta f+\eta \mathbf{1})=\gamma(f)$.

Proof.

For any $f \in \mathcal{F}, \theta>0, \eta \in \mathbb{R}_{+}$, the homogeneity of $I$ and Axiom (ADD) imply: $I(\theta f+\eta \mathbf{1})=$ $\theta I(f)+\eta$. In other words, $I$ is affine.

Next, let $\mu \in \mathbb{R}_{+}^{s}, \theta>0$ and $\eta \geq 0$, and define $f_{\mu} \in \mathcal{F}$ by $f_{\cdot i}=\mu$ for all $i \in K$. By definition of $I_{a}$, we have $I_{a}(\theta \mu+(\eta, \ldots, \eta))=I\left(\theta f_{\mu}+\eta \mathbf{1}\right)$. Because $I$ is affine, $I\left(\theta f_{\mu}+\eta \mathbf{1}\right)=\theta I\left(f_{\mu}\right)+\eta$. Finally, the definition of $I_{a}$ implies $I\left(f_{\mu}\right)=I_{a}(\mu)$. Therefore, $I_{a}(\theta \mu+(\eta, \ldots, \eta))=\theta I_{a}(\mu)+\eta$. In other words, $I_{a}: \mathbb{R}_{+}^{n} \rightarrow \mathbb{R}_{+}$is also affine. An analogous argument shows that $I_{p}: \mathbb{R}_{+}^{s} \rightarrow \mathbb{R}_{+}$ is affine. Thus, for all $f \in \mathcal{F}$,

$$
\left(I_{a} * I_{p}\right)(\theta f+\eta \mathbf{1})=I_{a}\left(I_{p}(\theta f+\eta \mathbf{1})\right)=I_{a}\left(\theta I_{p}(f)+(\eta, \ldots, \eta)\right)=\theta\left(I_{a} * I_{p}\right)(f)+\eta,
$$

where the first equality follows from the definition of $\left(I_{a} * I_{p}\right)$, the second equality is due to the fact that $I_{p}$ is affine, and the last equality follows from the fact that $I_{a}$ is affine. Therefore, $\left(I_{a} * I_{p}\right)$ is also affine. By a symmetric argument, one shows that $\left(I_{p} * I_{a}\right)$ is affine, too. 
Therefore, we can write:

$$
\begin{aligned}
I(\theta f+\eta \mathbf{1}) & =\gamma(\theta f+\eta \mathbf{1})\left(I_{p} * I_{a}\right)(\theta f+\eta \mathbf{1})+(1-\gamma(\theta f+\eta \mathbf{1}))\left(I_{a} * I_{p}\right)(\theta f+\eta \mathbf{1}) \\
& =\gamma(\theta f+\eta \mathbf{1})\left[\theta\left(I_{p} * I_{a}\right)(f)+\eta\right]+(1-\gamma(\theta f+\eta \mathbf{1}))\left[\theta\left(I_{a} * I_{p}\right)(f)+\eta\right] \\
& =\theta\left[\gamma(\theta f+\eta \mathbf{1})\left(I_{p} * I_{a}\right)(f)+(1-\gamma(\theta f+\eta \mathbf{1}))\left(I_{a} * I_{p}\right)(f)\right]+\eta .
\end{aligned}
$$

We can also write, however:

$$
I(\theta f+\eta \mathbf{1})=\theta I(f)+\eta=\theta\left[\gamma(f)\left(I_{p} * I_{a}\right)(f)+(1-\gamma(f))\left(I_{a} * I_{p}\right)(f)\right]+\eta .
$$

Comparing the two expressions for $I(\theta f+\eta \mathbf{1})$, we obtain:

$$
\begin{aligned}
& \theta\left[\gamma(f)\left(I_{p} * I_{a}\right)(f)+(1-\gamma(f))\left(I_{a} * I_{p}\right)(f)\right]+\eta \\
=\quad & \theta\left[\gamma(\theta f+\eta \mathbf{1})\left(I_{p} * I_{a}\right)(f)+(1-\gamma(\theta f+\eta \mathbf{1}))\left(I_{a} * I_{p}\right)(f)\right]+\eta .
\end{aligned}
$$

Assuming $\left(I_{p} * I_{a}\right)(f) \neq\left(I_{a} * I_{p}\right)(f)$, this implies $\gamma(\theta f+\eta \mathbf{1})=\gamma(f)$.

Claim 2. Let $f, g \in \mathcal{F}$. If $\left(I_{a} * I_{p}\right)(g)=\left(I_{a} * I_{p}\right)(f),\left(I_{p} * I_{a}\right)(f)=\left(I_{p} * I_{a}\right)(g)$, and $\left(I_{a} * I_{p}\right)(f) \neq$ $\left(I_{p} * I_{a}\right)(f)$ then $\gamma(f)=\gamma(g)$.

Proof.

By Axiom $(\mathrm{ADOM})$, if $\left(I_{a} * I_{p}\right)(g)=\left(I_{a} * I_{p}\right)(f)$ and $\left(I_{p} * I_{a}\right)(f)=\left(I_{p} * I_{a}\right)(g)$, then $I(f)=$ $I(g)$. Therefore: $\gamma(f)\left(I_{p} * I_{a}\right)(f)+(1-\gamma(f))\left(I_{a} * I_{p}\right)(f)=\gamma(g)\left(I_{p} * I_{a}\right)(g)+(1-\gamma(g))\left(I_{a} * I_{p}\right)(g)$, which implies $\gamma(f)=\gamma(g)$ since $\left(I_{a} * I_{p}\right)(g)=\left(I_{a} * I_{p}\right)(f),\left(I_{p} * I_{a}\right)(f)=\left(I_{p} * I_{a}\right)(g)$ and $\left(I_{a} * I_{p}\right)(f) \neq\left(I_{p} * I_{a}\right)(f)$.

Claim 3. Let $f, g \in \mathcal{F}$. If either $\left(I_{a} * I_{p}\right)(f)<\left(I_{p} * I_{a}\right)(f)$ and $\left(I_{a} * I_{p}\right)(g)<\left(I_{p} * I_{a}\right)(g)$, or $\left(I_{a} * I_{p}\right)(f)>\left(I_{p} * I_{a}\right)(f)$ and $\left(I_{a} * I_{p}\right)(g)>\left(I_{p} * I_{a}\right)(g)$ then $\gamma(f)=\gamma(g)$.

Proof.

Let $f, g \in \mathcal{F}$ be such that either $\left(I_{a} * I_{p}\right)(f)<\left(I_{p} * I_{a}\right)(f)$ and $\left(I_{a} * I_{p}\right)(g)<\left(I_{p} * I_{a}\right)(g)$, or $\left(I_{a} * I_{p}\right)(f)>\left(I_{p} * I_{a}\right)(f)$ and $\left(I_{a} * I_{p}\right)(g)>\left(I_{p} * I_{a}\right)(g)$. Let us define $h$ by:

$$
h=\frac{\left(I_{p} * I_{a}\right)(f)-\left(I_{a} * I_{p}\right)(f)}{\left(I_{p} * I_{a}\right)(g)-\left(I_{a} * I_{p}\right)(g)} g+\frac{\left(I_{p} * I_{a}\right)(g)\left(I_{a} * I_{p}\right)(f)-\left(I_{p} * I_{a}\right)(f)\left(I_{a} * I_{p}\right)(g)}{\left(I_{p} * I_{a}\right)(g)-\left(I_{a} * I_{p}\right)(g)} \mathbf{1}+\eta \mathbf{1},
$$

where $\eta>0$ is chosen such that $h \in \mathcal{F}$. 
Because $\left(I_{a} * I_{p}\right)$ is affine, we have:

$$
\begin{aligned}
\left(I_{a} * I_{p}\right)(h)= & \frac{\left(I_{p} * I_{a}\right)(f)-\left(I_{a} * I_{p}\right)(f)}{\left(I_{p} * I_{a}\right)(g)-\left(I_{a} * I_{p}\right)(g)}\left(I_{a} * I_{p}\right)(g) \\
& +\frac{\left(I_{p} * I_{a}\right)(g)\left(I_{a} * I_{p}\right)(f)-\left(I_{p} * I_{a}\right)(f)\left(I_{a} * I_{p}\right)(g)}{\left(I_{p} * I_{a}\right)(g)-\left(I_{a} * I_{p}\right)(g)}+\eta \\
= & \frac{\left(I_{p} * I_{a}\right)(g)\left(I_{a} * I_{p}\right)(f)-\left(I_{a} * I_{p}\right)(f)\left(I_{a} * I_{p}\right)(g)}{\left(I_{p} * I_{a}\right)(g)-\left(I_{a} * I_{p}\right)(g)}+\eta \\
= & \left(I_{a} * I_{p}\right)(f)+\eta .
\end{aligned}
$$

Similarly, one can show that $\left(I_{p} * I_{a}\right)(h)=\left(I_{p} * I_{a}\right)(f)+\eta$. Therefore, $\left(I_{a} * I_{p}\right)(h) \neq\left(I_{p} * I_{a}\right)(h)$ (recall that we assumed $\left.\left(I_{a} * I_{p}\right)(f) \neq\left(I_{p} * I_{a}\right)(f)\right)$. Claim 1 implies $\gamma(h)=\gamma(g)$. Since either $\left(I_{a} * I_{p}\right)(f)<\left(I_{p} * I_{a}\right)(f)$ or $\left(I_{a} * I_{p}\right)(f)>\left(I_{p} * I_{a}\right)(f)$, Claim 2 implies $\gamma(f)=\gamma(h)$. Hence, $\gamma(f)=\gamma(g) . \diamond$

Claim 3 implies that $\gamma(f)$ only depends on the ordering of $\left(I_{a} * I_{p}\right)(f)$ and $\left(I_{p} * I_{a}\right)(f)$, which completes the proof of the "necessary" part of the Theorem. ${ }^{11}$

We now turn to the "sufficiency" part of the Theorem. Because $I$ is clearly affine, Axiom (ADD) is satisfied. We now check axiom (ADOM). Let $f, g \in \mathcal{F}$ be such that $\left(I_{a} * I_{p}\right)(f) \geq$ $\left(I_{a} * I_{p}\right)(g)$ and $\left(I_{p} * I_{a}\right)(f) \geq\left(I_{p} * I_{a}\right)(g)$. Two cases may occur: either $(i) f$ and $g$ are evaluated with the same weights, or $(i i)$ they are evaluated with different weights. Case $(i)$ arises when $\left[\left(I_{a} * I_{p}\right)(f)-\left(I_{p} * I_{a}\right)(f)\right]\left[\left(I_{a} * I_{p}\right)(g)-\left(I_{p} * I_{a}\right)(g)\right] \geq 0$, whereas case $(i i)$ may arise when $\left[\left(I_{a} * I_{p}\right)(f)-\left(I_{p} * I_{a}\right)(f)\right]\left[\left(I_{a} * I_{p}\right)(g)-\left(I_{p} * I_{a}\right)(g)\right]<0$. Let us first consider case (i). Without loss of generality, let us assume that $\left(I_{a} * I_{p}\right)(f) \geq\left(I_{p} * I_{a}\right)(f)$. We then have: $I(f)=\alpha\left(I_{p} * I_{a}\right)(f)+(1-\alpha)\left(I_{a} * I_{p}\right)(f)$, and $I(g)=\alpha\left(I_{p} * I_{a}\right)(g)+(1-\alpha)\left(I_{a} * I_{p}\right)(g)$. Because, by assumption, $\left(I_{a} * I_{p}\right)(f) \geq\left(I_{a} * I_{p}\right)(g)$ and $\left(I_{p} * I_{a}\right)(f) \geq\left(I_{p} * I_{a}\right)(g)$, we get $I(f) \geq I(g)$, which implies that Axiom (ADOM) is satisfied.

Consider now case $(i i)$. Without loss of generality, assume that $\left(I_{a} * I_{p}\right)(f)>\left(I_{p} * I_{a}\right)(f)$ and $\left(I_{p} * I_{a}\right)(g)>\left(I_{a} * I_{p}\right)(g)$. We then have:

$$
I(f)=\alpha\left(I_{p} * I_{a}\right)(f)+(1-\alpha)\left(I_{a} * I_{p}\right)(f) \geq \alpha\left(I_{p} * I_{a}\right)(f)+(1-\alpha)\left(I_{p} * I_{a}\right)(f)=\left(I_{p} * I_{a}\right)(f),
$$

and:

$$
I(g)=\beta\left(I_{p} * I_{a}\right)(g)+(1-\beta)\left(I_{a} * I_{p}\right)(g) \leq \beta\left(I_{p} * I_{a}\right)(g)+(1-\beta)\left(I_{p} * I_{a}\right)(g)=\left(I_{p} * I_{a}\right)(g) .
$$

But we have, by assumption, $\left(I_{p} * I_{a}\right)(f) \geq\left(I_{p} * I_{a}\right)(g)$. Therefore, $I(f) \geq I(g)$, which implies that Axiom (ADOM) is satisfied.

\footnotetext{
${ }^{11}$ Uniqueness of $\alpha$ and $\beta$ directly follows from Theorem 2 .
} 


\section{Proof of Theorem 4 .}

The "if" part of the Theorem is straightforward. We hence only prove the "only if" part.

Since Axiom (GDOM) is satisfied, so is Axiom (ADOM). It follows from Theorem 3 that there exist $\alpha, \beta \in(0,1)$, such that:

$$
I(f)=\left\{\begin{array}{l}
\alpha\left(I_{p} * I_{a}\right)(f)+(1-\alpha)\left(I_{a} * I_{p}\right)(f), \text { if }\left(I_{a} * I_{p}\right)(f) \geq\left(I_{p} * I_{a}\right)(f) \\
\beta\left(I_{p} * I_{a}\right)(f)+(1-\beta)\left(I_{a} * I_{p}\right)(f), \text { if }\left(I_{p} * I_{a}\right)(f) \geq\left(I_{a} * I_{p}\right)(f)
\end{array}\right.
$$

where $I_{a}$ and $I_{p}$ are affine.

We want to prove that $\alpha=(1-\beta)$. Let $f \in \mathcal{F}$ be such that $\left(I_{a} * I_{p}\right)(f)>\left(I_{p} * I_{a}\right)(f)$. Now, let us define $h$ as follows: $h=-f+\left(I_{a} * I_{p}\right)(f) \mathbf{1}+\left(I_{p} * I_{a}\right)(f) \mathbf{1}+\eta \mathbf{1}$, where $\eta>0$ is chosen such that $h \in \mathcal{F}$. We can easily check that because $\left(I_{a} * I_{p}\right)$ and $\left(I_{p} * I_{a}\right)$ are affine (see the proof of Claim 1, Theorem 3), $\left(I_{a} * I_{p}\right)(h)=\left(I_{p} * I_{a}\right)(f)+\eta$ and $\left(I_{p} * I_{a}\right)(h)=\left(I_{a} * I_{p}\right)(f)+\eta$. Therefore, $\left(I_{p} * I_{a}\right)(h)>\left(I_{a} * I_{p}\right)(h)$, which entails:

$$
I(h)=\beta\left(I_{p} * I_{a}\right)(h)+(1-\beta)\left(I_{a} * I_{p}\right)(h)
$$

Now, define $g \in \mathcal{F}$ by $g=f+\eta \mathbf{1}$. We have, because $\left(I_{a} * I_{p}\right)$ and $\left(I_{p} * I_{a}\right)$ are affine: $\left(I_{a} * I_{p}\right)(g)=$ $\left(I_{a} * I_{p}\right)(f)+\eta=\left(I_{p} * I_{a}\right)(h)$ and $\left(I_{p} * I_{a}\right)(g)=\left(I_{p} * I_{a}\right)(f)+\eta=\left(I_{a} * I_{p}\right)(h)$. Therefore Axiom $(\mathrm{GDOM})$ implies that $I(g)=I(h)$. But because $\left(I_{a} * I_{p}\right)(g)>\left(I_{p} * I_{a}\right)(g)$, we have:

$$
I(g)=\alpha\left(I_{p} * I_{a}\right)(g)+(1-\alpha)\left(I_{a} * I_{p}\right)(g)=\alpha\left(I_{a} * I_{p}\right)(h)+(1-\alpha)\left(I_{p} * I_{a}\right)(h) .
$$

Therefore, we obtain:

$$
\beta\left(I_{p} * I_{a}\right)(h)+(1-\beta)\left(I_{a} * I_{p}\right)(h)=\alpha\left(I_{a} * I_{p}\right)(h)+(1-\alpha)\left(I_{p} * I_{a}\right)(h) .
$$

Finally, because $\left(I_{p} * I_{a}\right)(h)>\left(I_{a} * I_{p}\right)(h)$, equation (1) implies $\beta=(1-\alpha)$, which completes the proof. ${ }^{12}$

\section{Proof of Theorem 5.}

We first prove the "only if" part of the Theorem.

Claim 1. $I \in \mathcal{W}_{2} \cap \mathcal{W}_{3}$ and $I(f) \neq \frac{1}{2}\left(I_{p} * I_{a}\right)(f)+\frac{1}{2}\left(I_{a} * I_{p}\right)(f)$ implies that either $\left(I_{p} * I_{a}\right)(f) \geq$ $\left(I_{a} * I_{p}\right)(f)$ for all $f \in \mathcal{F}$, or $\left(I_{a} * I_{p}\right)(f) \geq\left(I_{p} * I_{a}\right)(f)$ for all $f \in \mathcal{F}$.

\section{Proof.}

\footnotetext{
${ }^{12}$ Uniqueness of $\delta$ directly follows from Theorem 2.
} 
Let us assume $I \in \mathcal{W}_{2} \cap \mathcal{W}_{3}$ and $I(f) \neq \frac{1}{2}\left(I_{p} * I_{a}\right)(f)+\frac{1}{2}\left(I_{a} * I_{p}\right)(f)$. In that case, there exist $\alpha$ and $\delta$ in $(0,1) \backslash\left\{\frac{1}{2}\right\}$, such that, for all $f \in \mathcal{F}$,

$$
I(f)=\alpha\left(I_{p} * I_{a}\right)(f)+(1-\alpha)\left(I_{a} * I_{p}\right)(f),
$$

and

$$
I(f)=\delta \min \left\{\left(I_{a} * I_{p}\right)(f),\left(I_{p} * I_{a}\right)(f)\right\}+(1-\delta) \max \left\{\left(I_{a} * I_{p}\right)(f),\left(I_{p} * I_{a}\right)(f)\right\},
$$

where (3) follows from Theorem 4. Let us assume that there exist $f$ and $g$ in $\mathcal{F}$, such that $\left(I_{a} * I_{p}\right)(f)>\left(I_{p} * I_{a}\right)(f)$ and $\left(I_{a} * I_{p}\right)(g)<\left(I_{p} * I_{a}\right)(g)$. Using equations $(2)$ and $(3),\left(I_{a} * I_{p}\right)(f)>$ $\left(I_{p} * I_{a}\right)(f)$ implies $\alpha=\delta$, whereas $\left(I_{a} * I_{p}\right)(g)<\left(I_{p} * I_{a}\right)(g)$ implies $\alpha=(1-\delta)$. But we assumed that $\alpha \neq \frac{1}{2}$ and $\delta \neq \frac{1}{2}$, which yields a contradiction. $\diamond$

Claim 2. If for all $f \in \mathcal{F},\left(I_{a} * I_{p}\right)(f) \leq\left(I_{p} * I_{a}\right)(f)$, then $I_{a}$ is the mathematical expectation with respect to a given probability distribution, or $I_{p}$ is a generalized minimum operator.

Proof.

We first introduce some notations, definitions, and a preliminary result due to Ghirardato, Klibanoff and Marinacci (1998).

We say that two vectors $\phi=\left(\phi_{1}, \ldots, \phi_{q}\right)$ and $\psi=\left(\psi_{1}, \ldots, \psi_{q}\right)$ of $\mathbb{R}^{q}$ are comonotonic if for every $i, j \in\{1, \ldots, q\},\left(\phi_{i}-\phi_{j}\right)\left(\psi_{i}-\psi_{j}\right) \geq 0$. If there exist $\alpha \geq 0$ and $\beta \in \mathbb{R}$ such that either $\phi_{i}=\alpha \psi_{i}+\beta$ for all $i$, or $\psi_{i}=\alpha \phi_{i}+\beta$ for all $i$, or both, we say that $\phi$ and $\psi$ are affinely related. Finally, let $\mathcal{C}$ be the set of compact and convex sets of probability measures over $\mathbb{R}^{q}$.

Affinely related vectors are important, because the min-of-means functionals are additive for affinely related vectors. More precisely, we have the following result, due to Ghirardato, Klibanoff and Marinacci (1998) (Theorem 1 and Lemma 1).

Proposition (Ghirardato, Klibanoff and Marinacci (1998))

Let $J_{C}$ denote the min-of-means functional defined on $\mathbb{R}^{q}$, with respect to the set of probability measures $C \in \mathcal{C}$. Then the following two statements are equivalent:

(i) $\phi$ and $\psi$ in $\mathbb{R}^{q}$ are affinely related;

(ii) $J_{C}(\phi+\psi)=J_{C}(\phi)+J_{C}(\psi)$ for all $C \in \mathcal{C}$.

Moreover, for a given $C \in \mathcal{C}$, the following two statements are equivalent:

(iii) $J_{C}(\phi+\psi)=J_{C}(\phi)+J_{C}(\psi)$;

(iv) $\left(\arg \min _{p \in C} p \cdot \phi\right) \cap\left(\arg \min _{p \in C} p \cdot \psi\right) \neq \emptyset$. 
We can now turn to the proof of the claim. Assume that for all $f \in \mathcal{F},\left(I_{a} * I_{p}\right)(f) \leq\left(I_{p} * I_{a}\right)(f)$ and that $I_{p}$ is not a generalized minimum operator. We are going to show that $I_{a}$ is then necessarily the mathematical expectation with respect to a given probability distribution in $\mathcal{P}_{S}$ (i.e., $C_{I_{a}}$ is a singleton).

Because $I_{p}$ is not a generalized minimum operator, for all $\left\{i_{1}, \ldots, i_{k}\right\} \subseteq K, C_{I_{p}} \neq c o\left(e_{i_{1}}, \ldots, e_{i_{k}}\right)$, where for all $i \in K, e_{i} \in \mathbb{R}^{n}$ is such that its $i^{\text {th }}$ entry is equal to 1 , whereas all its other entries are equal to zero. ${ }^{13}$

Let $K_{1}=\left\{i \in K \mid e_{i} \notin C_{I_{p}}\right\}$. By construction, $K_{1} \neq \emptyset$, otherwise we would have $C_{I_{p}}=$ $c o\left(e_{1}, \ldots, e_{K}\right)$. Thus, it is possible to define $\bar{p}=\max _{\left\{p \in C_{I_{p}}, i \in K_{1}\right\}} p_{i}$. The $\bar{p}$ parameter represents the largest weight given by elements of $C_{I_{p}}$ to elements of $K_{1}$.

We are going to prove that $0<\bar{p}<1$, and construct a vector $\lambda$ such that $I_{p}(\lambda)$ depends on $\bar{p}$.

By definition of $K_{1}$, any $i \in K_{1}$ is such that $p_{i}<1$ for any $p \in C_{I_{p}}$. Thus, $\bar{p}<1$. Furthermore, there exists at least one $i \in K_{1}$ and one $p \in C_{I_{p}}$ such that $p_{i} \neq 0$ (otherwise we would have $\left.C_{I_{p}}=c o\left(e_{i}\right)_{i \notin K_{1}}\right)$. Thus, $\bar{p}>0$.

Now, consider any $j_{0}$ in $K_{1}$ such that there exists $\tilde{p}$ in $C_{I_{p}}$ with $\tilde{p}_{j_{0}}=\bar{p}$. By construction, we have $\bar{p}=\max _{\left\{p \in C_{I_{p}}\right\}} p_{j_{0}}$. Define $\lambda \in \mathbb{R}^{n}$ by $\lambda_{i}=1$ for all $i \neq j_{0}$, and $\lambda_{j_{0}}=0$. Also define $C_{j_{0}}=\left\{p \in C_{I_{p}} \mid p_{j_{0}}=\bar{p}\right\}$. Given the definition of $\lambda, p \cdot \lambda=\left(1-p_{j_{0}}\right)$ for any $p$ in $C_{I_{p}}$ and therefore:

$$
\arg \min _{p \in C_{I_{p}}} p \cdot \lambda=\arg \min _{p \in C_{I_{p}}}\left(1-p_{j_{0}}\right)=\arg \max _{p \in C_{I_{p}}} p_{j_{0}} .
$$

But, because $\bar{p}=\max _{\left\{p \in C_{I_{p}}\right\}} p_{j_{0}}$, any $p \in \arg \max _{p \in C_{I_{p}}} p_{j_{0}}$ satisfies $p_{j_{0}}=\bar{p}$. Thus we have $\arg \min _{p \in C_{I_{p}}} p \cdot \lambda \subseteq C_{j_{0}}$.

Now, assume that there exist $\tilde{\mu}, \bar{\mu} \in \mathbb{R}_{+}^{s}$, such that:

$$
\left(\arg \min _{q \in C_{I_{a}}} q \cdot \bar{\mu}\right) \cap\left(\arg \min _{q \in C_{I_{a}}} q \cdot \tilde{\mu}\right)=\emptyset .
$$

As discussed below, this amounts to assuming that $I_{a}$ is not an expectation operator. Note that this assumption implies that neither $\tilde{\mu}$ nor $\bar{\mu}$ is a vector of zeros. Using $\bar{p}$ and $\lambda$ we are going to show that such an hypothesis contradicts the assumption that $\left(I_{a} * I_{p}\right)(f) \leq\left(I_{p} * I_{a}\right)(f)$ for all $f \in \mathcal{F}$.

Note that for all $\theta>0, \arg \min _{q \in C_{I_{a}}} q \cdot \tilde{\mu}=\arg \min _{q \in C_{I_{a}}} q \cdot(\theta \tilde{\mu})$. Let $\theta>0$ be such that $\theta \tilde{\mu}_{\sigma}>\bar{\mu}_{\sigma}$ for all $\sigma \in S$, and define $\mu=\theta \tilde{\mu}$. We have $\mu_{\sigma}>\bar{\mu}_{\sigma}$ for all $\sigma \in S$ and $\left(\arg \min _{q \in C_{I_{a}}} q \cdot \bar{\mu}\right) \cap\left(\arg \min _{q \in C_{I_{a}}} q \cdot \mu\right)=\emptyset$.

\footnotetext{
${ }^{13}$ If $\lambda$ and $\lambda^{\prime}$ are two vectors in $\mathbb{R}_{+}^{n}, \cos \left(\lambda, \lambda^{\prime}\right)$ denotes the convex hull of $\lambda$ and $\lambda^{\prime}$, i.e., $\operatorname{co}\left(\lambda, \lambda^{\prime}\right)=$ $\left\{\tilde{\lambda} \in \mathbb{R}_{+}^{n} \mid \exists \alpha \in[0,1]\right.$ s.t. $\left.\tilde{\lambda}=\alpha \lambda+(1-\alpha) \lambda^{\prime}\right\}$.
} 
Let $f \in \mathcal{F}$ be defined by $f_{\cdot i}=\mu$ if $i \neq j_{0}$, and $f_{\cdot j_{0}}=\bar{\mu}$. For all $\sigma \in S, f_{\sigma i}=\mu_{\sigma}$ if $i \neq j_{0}$ and $f_{\sigma j_{0}}=\bar{\mu}_{\sigma}$. Therefore, $f_{\sigma i}=\left(\mu_{\sigma}-\bar{\mu}_{\sigma}\right) \lambda_{i}+\bar{\mu}_{\sigma}$, which means that $f_{\sigma}$. and $\lambda$ are affinely related for all $\sigma \in S$. Given this fact, the first part of the Proposition (i.e., $(i) \Rightarrow(i i)$ ) implies that $I_{p}\left(f_{\sigma}+\lambda\right)=I_{p}\left(f_{\sigma}\right)+I_{p}(\lambda)$. Given this equality, the second part of the proposition (i.e., $($ iii $) \Rightarrow($ iv $))$ implies that:

$$
\left(\arg \min _{\tilde{p} \in C_{I_{p}}} \tilde{p} \cdot \lambda\right) \cap\left(\arg \min _{\tilde{p} \in C_{I_{p}}} \tilde{p} \cdot f_{\sigma} \cdot\right) \neq \emptyset .
$$

Thus, given that $\arg \min _{p \in C_{I_{p}}} p \cdot \lambda \subseteq C_{j_{0}}$, there exists $p^{*} \in C_{j_{0}}$ such that $I_{p}\left(f_{\sigma}.\right)=p^{*} \cdot f_{\sigma}$, which implies $I_{p}\left(f_{\sigma \cdot}\right)=(1-\bar{p}) \mu_{\sigma}+\bar{p} \bar{\mu}_{\sigma}$.

Now, let $r \in \arg \min _{\tilde{r} \in C_{I_{a}}} \tilde{r} \cdot I_{p}(f)$. We have then, by definition of $I_{a},\left(I_{a} * I_{p}\right)(f)=r \cdot I_{p}(f)$, i.e.,

$$
\left(I_{a} * I_{p}\right)(f)=\sum_{\sigma} r_{\sigma}\left[(1-\bar{p}) \mu_{\sigma}+\bar{p} \bar{\mu}_{\sigma}\right]
$$

which can be written as:

$$
\left(I_{a} * I_{p}\right)(f)=(1-\bar{p}) \sum_{\sigma} r_{\sigma} \mu_{\sigma}+\bar{p} \sum_{\sigma} r_{\sigma} \bar{\mu}_{\sigma} .
$$

Next, observe that $I_{a}\left(f_{\cdot i}\right)=I_{a}(\mu)$ for all $i \neq j_{0}$ and $I_{a}\left(f_{\cdot j_{0}}\right)=I_{a}(\bar{\mu})$. Furthermore, given that $\mu_{\sigma}>\bar{\mu}_{\sigma}$ for all $\sigma \in S$, we have $I_{a}(\mu)>I_{a}(\bar{\mu})$. But it is easily checked that $I_{a}\left(f_{\cdot i}\right)=\left(I_{a}(\mu)-\right.$ $\left.I_{a}(\bar{\mu})\right) \lambda_{i}+I_{a}(\bar{\mu})$, which means that $I_{a}(f)$ and $\lambda$ are affinely related. Therefore, using the same arguments as above, there exists $\hat{p} \in C_{j_{0}}$ such that $\left(I_{p} * I_{a}\right)(f)=\hat{p} \cdot I_{a}(f)=(1-\bar{p}) I_{a}(\mu)+\bar{p} I_{a}(\bar{\mu})$.

Now, by definition of $I_{a}$, and since $r \in C_{I_{a}}$, we have $I_{a}\left(f_{\cdot i}\right) \leq r \cdot f_{\cdot i}$ for all $i \in K$. If none of these inequalities were strict, we would have $r \in \arg \min _{\tilde{r} \in C_{I_{a}}} \tilde{r} \cdot f_{\cdot i}$ for all $i \in K$, which would contradict $\left(\arg \min _{q \in C_{I_{a}}} q \cdot \bar{\mu}\right) \cap\left(\arg \min _{q \in C_{I_{a}}} q \cdot \mu\right)=\emptyset$. Thus, at least one of these inequalities is strict. This implies (recall that $0<\bar{p}<1$ ):

$$
\left(I_{p} * I_{a}\right)(f)=(1-\bar{p}) I_{a}(\mu)+\bar{p} I_{a}(\bar{\mu})<(1-\bar{p})(r \cdot \mu)+\bar{p}(r \cdot \bar{\mu}) .
$$

But we have:

$$
\begin{aligned}
(1-\bar{p})(r \cdot \mu)+\bar{p}(r \cdot \bar{\mu}) & =(1-\bar{p}) \sum_{\sigma} r_{\sigma} \mu_{\sigma}+\bar{p} \sum_{\sigma} r_{\sigma} \bar{\mu}_{\sigma} \\
& =\left(I_{a} * I_{p}\right)(f),
\end{aligned}
$$

where the last equality follows from equation (4). Therefore, $\left(I_{p} * I_{a}\right)(f)<\left(I_{a} * I_{p}\right)(f)$, a contradiction. Therefore, for all $\mu, \bar{\mu} \in \mathbb{R}_{+}^{s},\left(\arg \min _{q \in C_{I_{a}}} q \cdot \mu\right) \cap\left(\arg \min _{q \in C_{I_{a}}} q \cdot \bar{\mu}\right) \neq \emptyset$. By the Proposition, this implies that $I_{a}$ is additive on $\mathbb{R}_{+}^{s}$, which implies that $I_{a}$ is the expectation with respect to a given probability distribution. 
Claim 3. If for all $f \in \mathcal{F},\left(I_{a} * I_{p}\right)(f) \geq\left(I_{p} * I_{a}\right)(f)$, then $I_{p}$ is the mathematical expectation with respect to a given probability distribution, or $I_{a}$ is a generalized minimum operator.

Proof.

By symmetry, the proof is similar to the proof of Claim $2 . \diamond$

The "only if" part of the Theorem follows from Claims 1 to 3.

We now turn to the "if" part of the Theorem. In what follows, we assume that $I \in \mathcal{W}_{3}$.

First, assume that $I_{a}$ is the mathematical expectation with respect to a given probability measure $q \in \mathcal{P}_{S}$. Then, for all $f \in \mathcal{F}, I_{a}(f)=\left(q \cdot f_{\cdot 1}, \ldots, q \cdot f_{\cdot s}\right)=\sum_{\sigma} q_{\sigma} f_{\sigma \cdot}$, and therefore $\left(I_{p} * I_{a}\right)(f)=I_{p}\left(\sum_{\sigma} q_{\sigma} f_{\sigma}.\right)$. On the other hand, $\left(I_{a} * I_{p}\right)(f)=q \cdot I_{p}(f)=\sum_{\sigma} q_{\sigma} I_{p}\left(f_{\sigma}.\right)$. But any min-of-means functional is concave (see Gilboa and Schmeidler (1989)). Therefore, by Jensen's inequality: $\sum_{\sigma} q_{\sigma} I_{p}\left(f_{\sigma}.\right) \leq I_{p}\left(\sum_{\sigma} q_{\sigma} f_{\sigma}.\right)$, and therefore $\left(I_{a} * I_{p}\right)(f) \leq\left(I_{p} * I_{a}\right)(f)$ for all $f \in \mathcal{F}$, which clearly implies that $I \in \mathcal{W}_{2}$.

By symmetry, if $I_{p}$ is the mathematical expectation with respect to a given probability measure, the same argument leads to $\left(I_{a} * I_{p}\right)(f) \geq\left(I_{p} * I_{a}\right)(f)$ for all $f \in \mathcal{F}$, which clearly implies that $I \in \mathcal{W}_{2}$.

Now, assume that $I_{p}$ is a generalized minimum operator. Then, there exists $K_{0}=\left\{i_{1}, \ldots, i_{k}\right\} \subseteq$ $K$ such that $C_{I_{p}}=c o\left(e_{i}\right)_{i \in K_{0}}$. In this case, for all $f \in \mathcal{F}$, and all $\sigma \in S, I_{p}\left(f_{\sigma}.\right)=\min _{i \in K_{0}} f_{\sigma i}$. Therefore, $\left(I_{a} * I_{p}\right)(f)=I_{a}\left(\min _{i \in K_{0}} f_{1 i}, \ldots, \min _{i \in K_{0}} f_{s i}\right)$, from which it follows by monotonicity of $I_{a}$ that for all $j \in K_{0},\left(I_{a} * I_{p}\right)(f) \leq I_{a}\left(f_{\cdot j}\right)$. But $\left(I_{p} * I_{a}\right)(f)=\min _{i \in K_{0}} I_{a}\left(f_{\cdot i}\right)$. Therefore, there exists $j_{0} \in K_{0}$ such that $\left(I_{p} * I_{a}\right)(f)=I_{a}\left(f_{\cdot j_{0}}\right)$. Choosing $j=j_{0}$, it follows that $\left(I_{a} * I_{p}\right)(f) \leq\left(I_{p} * I_{a}\right)(f)$, for all $f \in \mathcal{F}$, which implies that $I \in \mathcal{W}_{2}$.

By symmetry, if $I_{a}$ is a generalized minimum operator, the same argument leads to $\left(I_{a} *\right.$ $\left.I_{p}\right)(f) \geq\left(I_{p} * I_{a}\right)(f)$ for all $f \in \mathcal{F}$, which clearly implies that $I \in \mathcal{W}_{2}$.

Finally, if $I(f)=\frac{1}{2}\left(I_{p} * I_{a}\right)(f)+\frac{1}{2}\left(I_{a} * I_{p}\right)(f)$ for all $f \in \mathcal{F}$, then trivially $I \in \mathcal{W}_{2}$.

Proof of Theorem 6.

The "if" part of the Theorem is straightforward. We hence only prove the "only if" part.

Since $I \in \mathcal{W}$ and $I$ satisfies Axiom (ADD), $I_{a}$ and $I_{p}$ are affine.

Let $f \in \mathcal{F}$ be such that $\left(I_{p} * I_{a}\right)(f) \neq\left(I_{a} * I_{p}\right)(f)$ and consider $g$ defined by: $g_{\sigma i}=$ $\frac{1}{2} I_{a}\left(f_{\cdot i}\right)+\frac{1}{2} I_{p}\left(f_{\sigma}.\right)$, for all $i$ in $K$ and all $\sigma$ in $S$. We then obtain:

$$
I_{a}\left(g_{\cdot i}\right)=I_{a}\left(f_{\cdot i}\right)+\left[\frac{1}{2}\left(I_{a} * I_{p}\right)(f)-\frac{1}{2} I_{a}\left(f_{\cdot i}\right)\right], \forall i \in K,
$$


and:

$$
I_{p}\left(g_{\sigma} .\right)=I_{p}\left(f_{\sigma}\right)+\left[\frac{1}{2}\left(I_{p} * I_{a}\right)(f)-\frac{1}{2} I_{p}\left(f_{\sigma}\right)\right], \forall \sigma \in S .
$$

Now, let us define $u \in \mathbb{R}^{n}$ by $u_{i}=\frac{1}{2}\left(I_{a}\left(f_{\cdot i}\right)-\left(I_{a} * I_{p}\right)(f)\right)$, for all $i$ in $K$, and $v \in \mathbb{R}^{s}$ by $v_{\sigma}=\frac{1}{2}\left(\left(I_{p} * I_{a}\right)(f)-I_{p}\left(f_{\sigma}.\right)\right)$. We hence have: $I_{a}(g)=I_{a}(f)-u$ and $I_{p}(g)=I_{p}(f)+v$. Finally, let $k>0$ be large enough to have $\mathbf{u}_{\sigma}+\mathbf{k}$ and $\mathbf{v}_{\cdot i}+\mathbf{k}$ in $\mathcal{F}$, where $\mathbf{u}_{\sigma}+\mathbf{k}\left(\mathbf{v}_{\cdot i}+\mathbf{k}\right)$ represents the matrix with all rows (columns) equal to $u+k \mathbf{1}_{n}\left(v+k \mathbf{1}_{s}\right) .{ }^{14}$

Without loss of generality, we assume that $I$ is normalized with $I(\mathbf{1})=1$. Then, we can easily check that, since $I_{a}$ and $I_{p}$ are affine, $\left(I_{a} * I_{p}\right)\left(\mathbf{u}_{\sigma}+\mathbf{k}\right)=\left(I_{p} * I_{a}\right)\left(\mathbf{u}_{\sigma}+\mathbf{k}\right)=\frac{1}{2}\left[\left(I_{p} * I_{a}\right)(f)-\right.$ $\left.\left(I_{a} * I_{p}\right)(f)\right]+k$, which implies, since $I$ is a WCI functional, that $I\left(\mathbf{u}_{\sigma} .+\mathbf{k}\right)=\frac{1}{2}\left[\left(I_{p} * I_{a}\right)(f)-\right.$ $\left.\left(I_{a} * I_{p}\right)(f)\right]+k$. Similarly, $\left(I_{a} * I_{p}\right)\left(\mathbf{v}_{\cdot i}+\mathbf{k}\right)=\left(I_{p} * I_{a}\right)\left(\mathbf{v}_{\cdot i}+\mathbf{k}\right)=\frac{1}{2}\left[\left(I_{p} * I_{a}\right)(f)-\left(I_{a} * I_{p}\right)(f)\right]+k$. Therefore, $I\left(\mathbf{v}_{\cdot i}+\mathbf{k}\right)=\frac{1}{2}\left[\left(I_{p} * I_{a}\right)(f)-\left(I_{a} * I_{p}\right)(f)\right]+k$, from which it follows that $I\left(\mathbf{u}_{\sigma} .+\mathbf{k}\right)=$ $I\left(\mathbf{v}_{\cdot i}+\mathbf{k}\right)$. Therefore, $v \in \mathcal{S}(u)$. Hence, by Axiom (SYM), we have $f \sim g$. Because $I_{a}$ and $I_{p}$ are affine, however, we obtain, using equations (5) and (6):

$$
\begin{aligned}
\left(I_{p} * I_{a}\right)(g) & =\left(I_{p} * I_{a}\right)(f)+\frac{1}{2}\left(I_{a} * I_{p}\right)(f)-\frac{1}{2}\left(I_{p} * I_{a}\right)(f) \\
& =\frac{1}{2}\left(I_{p} * I_{a}\right)(f)+\frac{1}{2}\left(I_{a} * I_{p}\right)(f),
\end{aligned}
$$

and:

$$
\begin{aligned}
\left(I_{a} * I_{p}\right)(g) & =\left(I_{a} * I_{p}\right)(f)+\frac{1}{2}\left(I_{p} * I_{a}\right)(f)-\frac{1}{2}\left(I_{a} * I_{p}\right)(f) \\
& =\frac{1}{2}\left(I_{p} * I_{a}\right)(f)+\frac{1}{2}\left(I_{a} * I_{p}\right)(f) .
\end{aligned}
$$

Hence, by Theorem 1, $I(g)=\frac{1}{2}\left(I_{a} * I_{p}\right)(f)+\frac{1}{2}\left(I_{p} * I_{a}\right)(f)$. Therefore, $I(f)=\frac{1}{2}\left(I_{a} * I_{p}\right)(f)+$ $\frac{1}{2}\left(I_{p} * I_{a}\right)(f)$. We can then conclude that, for all $f$ such that $\left(I_{a} * I_{p}\right)(f) \neq\left(I_{p} * I_{a}\right)(f), \gamma(f)=\frac{1}{2}$.

Finally, if $\left(I_{a} * I_{p}\right)(f)=\left(I_{p} * I_{a}\right)(f)$, one obviously get $I(f)=\frac{1}{2}\left(I_{a} * I_{p}\right)(f)+\frac{1}{2}\left(I_{p} * I_{a}\right)(f)$.

\section{References}

Atkinson, A. B. (1970): "On the Measurement of Inequality," Journal of Economic Theory, $2,244-263$.

Ben Porath, E., I. Gilboa, and D. Schmeidler (1997): "On the Measurement of Inequality under Uncertainty," Journal of Economic Theory, 75, 194-204.

\footnotetext{
${ }^{14} k$ should be chosen large enough to have $\frac{1}{2}\left(I_{a} * I_{p}\right)(f)-\frac{1}{2} I_{a}\left(f_{\cdot i}\right)+k>0$ for all $i$, and $\frac{1}{2}\left(I_{p} * I_{a}\right)(f)-\frac{1}{2} I_{p}\left(f_{\sigma}\right)+k>$ 0 for all $\sigma$.
} 
Broome, J. (1984): "Uncertainty and Fairness," The Economic Journal, 94, 624-632.

Debreu, G. (1959): Theory of value. Wiley, New York.

Diamond, P. (1967): "Cardinal Welfare, Individualistic Ethics, and Interpersonal Comparison of Utility : Comment," Journal of Political Economy, 75, 765-766.

Ghirardato, P., P. Klibanoff, and M. Marinacci (1998): "Additivity with multiple priors," Journal of Mathematical Economics, 30, 405-420.

Gilboa, I., And D. Schmeidler (1989): "Maximin Expected Utility With a Non-Unique Prior," Journal of Mathematical Economics, 18, 141-153.

Hammond, P. J. (1981): "Ex-ante and ex-post welfare optimality under uncertainty," Economica, 48, 235-350.

Kolm, S.-C. (1976): "Unequal Inequalities. I," Journal of Economic Theory, 12, 416-442.

Myerson, R. B. (1981): "Utlitarianism, egalitarianism, and the timing effect in social choice problems," Econometrica, 49, 883-897.

Sen, A. K. (1973): On Economic Inequality. Clarendon Press, Oxford.

YAari, M., AND M. BAR-Hillel (1984): "On Dividing Justly," Social Choice and Welfare, 1, $1-24$. 